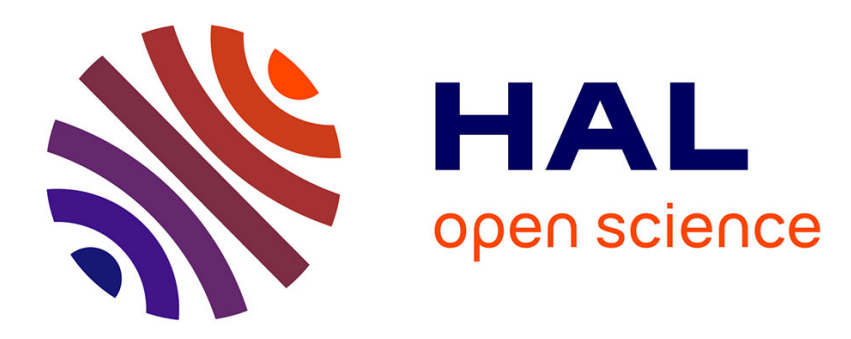

\title{
H-convergence and numerical schemes for elliptic equations
}

Robert Eymard, Thierry Gallouët

\section{To cite this version:}

Robert Eymard, Thierry Gallouët. H-convergence and numerical schemes for elliptic equations. SIAM Journal on Numerical Analysis, 2003, Volume 41, Number 2, pp.539-562. hal-00003332

\section{HAL Id: hal-00003332 \\ https://hal.science/hal-00003332}

Submitted on 25 Nov 2004

HAL is a multi-disciplinary open access archive for the deposit and dissemination of scientific research documents, whether they are published or not. The documents may come from teaching and research institutions in France or abroad, or from public or private research centers.
L'archive ouverte pluridisciplinaire HAL, est destinée au dépôt et à la diffusion de documents scientifiques de niveau recherche, publiés ou non, émanant des établissements d'enseignement et de recherche français ou étrangers, des laboratoires publics ou privés. 


\title{
H-convergence and numerical schemes for elliptic problems
}

\author{
R. Eymard*, T. Gallouët ${ }^{\dagger}$
}

November 25, 2004

\begin{abstract}
We study the convergence of two coupled numerical schemes, which are a discretization of a so-called elliptic-hyperbolic system. Only weak convergence properties are proved on the discrete diffusion of the elliptic problem and an adaptation of the H-convergence method gives a convergence property of the elliptic part of the scheme. The limit of the approximate solution is then the solution of an elliptic problem, the diffusion of which is not in the general case the H-limit of the discrete diffusion. In a particular case, a kind of weak limit is then obtained for the hyperbolic equation.
\end{abstract}

AMS Subject Classifications: 35K65, 35K55.

Keywords: H-convergence, finite volume schemes, two-phase flow, porous media.

\section{Introduction}

Numerical simulation takes an important place in the oil recovery engineering. In many cases, the engineer should represent at the same time the thermodynamical evolution of the hydrocarbon components during the pressure drop due to the extraction of oil, and the mass transfers in the oil reservoir. In this paper, we focus on the consequences of a mobility contrast between an injected fluid (generally water) and the oil in place, in a very simple case: oil and water are assumed to be incompressible immiscible fluid phases with a common pressure, and the reservoir is supposed to be a horizontal homogeneous isotropic domain. Following [3], the conservation equations for such a two-phase flow in this particular case, using Darcy's law, can be written as

$$
\left.\begin{array}{ll}
\frac{\partial s}{\partial t}-\operatorname{div}(\gamma(s) \lambda(s) \nabla u) & =(\bar{f})^{+} \gamma(\bar{s})-(\bar{f})^{-} \gamma(s), \\
\frac{\partial(1-s)}{\partial t}-\operatorname{div}((1-\gamma(s)) \lambda(s) \nabla u) & =(\bar{f})^{+}(1-\gamma(\bar{s}))-(\bar{f})^{-}(1-\gamma(s))
\end{array}\right\} \text { in } \Omega,
$$

with the boundary conditions

$$
\begin{aligned}
& u=0 \text { on } \partial \Omega \times \mathbb{R}_{+} \\
& s=\hat{s} \text { on }\left\{(x, t) \in \partial \Omega \times \mathbb{R}_{+}, \nabla u(x, t) \cdot \mathbf{n}_{\partial \Omega}(x) \geq 0\right\}
\end{aligned}
$$

In (1) and (2), the domain $\Omega$ represents the porous medium, $u$ represents the common pressure of the two phases, $s$ represents the saturation of the water phase, $\gamma(s)$ is a nondecreasing function which is called the "fractional flow", with $\gamma(0)=0$ and $\gamma(1)=1$, the positive function $\lambda(s)$ is the "total mobility" of the two phases (the sum of the mobility of water and the mobility of oil), the function $\bar{f}(x, t)$ represents the rates at the wells, $\bar{s}(x, t)$ is the saturation of the injected fluids (the injected rate corresponds to the positive part of the function $\bar{f}$, the produced rate to the negative part, and the repartition of the production between water and oil is determined by the saturation in the reservoir), the function $\hat{s}(x, t)$ is the saturation of incoming fluids at the boundary. We denote, for all real value $z, z^{+}=\max (z, 0)$ and $z^{-}=\max (-z, 0)$.

\footnotetext{
*Université de Marne-la-Vallée, 77454 Marne-la-vallée Cedex 2, France, eymard@math.univ-mlv.fr

†Université Aix-Marseille 1, 13453 Marseille Cedex 13, France, gallouet@cmi.univ-mrs.fr
} 
The existence of a solution to (1) is an open problem if the function $\lambda$ is not reduced to a constant. A number of numerical schemes for this problem have already been discussed in the literature. Nevertheless, their convergence has only recently be studied in the only case of a constant function $\lambda$ : the convergence of a numerical scheme involving a finite volume method for the computation of the saturation $s$ and a standard finite element for the computation of the pressure $u$ is proved in [7], whereas a convergence proof for a finite volume method for the discretization of both equations is presented in [21] and a convergence proof for a mixed finite element - finite volume scheme is given in [15].

The objective of this paper is the study of the convergence properties of finite volume methods in the case where the function $\lambda$ is not a constant function. This problem appears to be very close to the study of the convergence, when $\varepsilon \longrightarrow 0$ of the solution of the problem

$$
\left.\begin{array}{ll}
\frac{\partial s_{\varepsilon}}{\partial t}-\operatorname{div}\left(\gamma\left(s_{\varepsilon}\right) \lambda\left(s_{\varepsilon}\right) \nabla u_{\varepsilon}\right)-\varepsilon \Delta s_{\varepsilon} & =(\bar{f})^{+} \gamma(\bar{s})-(\bar{f})^{-} \gamma\left(s_{\varepsilon}\right), \\
\frac{\partial\left(1-s_{\varepsilon}\right)}{\partial t}-\operatorname{div}\left(\left(1-\gamma\left(s_{\varepsilon}\right)\right) \lambda\left(s_{\varepsilon}\right) \nabla u_{\varepsilon}\right)+\varepsilon \Delta s_{\varepsilon} & =(\bar{f})^{+}(1-\gamma(\bar{s}))-(\bar{f})^{-}\left(1-\gamma\left(s_{\varepsilon}\right)\right)
\end{array}\right\} \text { in } \Omega
$$

where the additional term $\varepsilon \Delta s_{\varepsilon}$ stands for a diffusive term, which is similar to the diffusion added by the upstream weighted numerical schemes. Such a diffusive term is slightly different from that which comes from the introduction of a capillary pressure term, yielding some degeneration similar to that of the porous media equation (see [1], [2], [11] and [5] for the existence of a solution of the continuous problem and see [10] for the proof of the convergence of a finite volume scheme).

In order to make clear the tools that appear, we shall consider a steady-state version of (1) (see (50) below). The main result of this paper is the proof that, using a coupled finite volume scheme for the approximation of this system of equations, the approximate pressure converges in $L^{2}(\Omega)$ to the solution of an elliptic problem whose coefficients are obtained by the same method as the classical H-convergence proof (following [17], [14] or [19]) whereas the approximate saturation only converges in a weak sense (namely in $L^{\infty}(\Omega)$ for the weak $\star$ topology). The use, in the discrete setting, of a notion similar to $\mathrm{H}$-convergence is natural: indeed, the existence of a limit as $\varepsilon \longrightarrow 0$ to the family of pressures $\left(u_{\varepsilon}\right)_{\varepsilon>0}$, solution to the sum of a steady version of the equations (3), immediately results from H-convergence (see Section 2). Note that an extension of the H-convergence background to a discrete setting has been performed, see [16] and mainly [12] for the proof of the existence of a "H-limit" to a subsequence of a sequence of discrete elliptic operators, using regular structured grids and finite differences. The objective is here to study the limit of a sequence of finite volume approximations on general meshes, whereas the discrete diffusion results from the coupling of the two discrete conservation equations. The fact that the two unknowns are computed in the same grids makes different, in the general case, the notion of continuous and discrete $\mathrm{H}$-limits, which suggests to distinguish the vocabulary devoted to both notions. It is also interesting to notice that the question of the independence of these limit coefficients on the way that some diffusion is added in (1) is not known. It is however clear that the limit $(u, s)$ of the numerical scheme or of the parabolic regularization (namely (3)) is a solution of (1) if a strong convergence result can be proved for the saturation. This sufficient condition seems to be necessary for a large class of data: for example, it is already necessary in the case of a constant function $\lambda$ when the function $\gamma$ is genuinely nonlinear.

This paper is organized as follows:

- in Section 2, a short review of the concept of H-convergence is made, and some examples of application of this notion are given,

- in Section 3, results are recalled on finite volume methods for elliptic problems,

- in Section 4, an adaptation of H-convergence to the study of the convergence of numerical schemes for elliptic problems is made,

- the convergence study of the coupled scheme for the two-phase flow problem is done in Section 5,

- some concluding remarks give guidelines for further works. 


\section{Some results of $\mathrm{H}$-convergence}

The notion of H-convergence is used for the physical description of effective properties, at the macroscopic level, of heterogeneous materials in which some diffusive phenomena occur. The assumption which is then done is that the scale of the heterogenities is small compared to the macroscopic scale. Let us take the example of the Dirichlet problem, which models for example the steady flow of a monophasic incompressible fluid in a heterogeneous porous medium, using Darcy's law. We assume that the pressure of the fluid is constant at the boundary of the domain and that some volumic source terms represent the injection and the production of fluid throughout some wells. The question of the existence of an "effective" permeability field, which could allow the computation of accurate approximate solution using only a coarse discretization (which means a discretization at the macroscopic scale) is of major interest for the industrial applications; this question can, in some cases, be handled using the notion of $\mathrm{H}$-convergence.

\subsection{Notations for the Dirichlet problem}

Let $\Omega$ be an open bounded subset of $\mathbb{R}^{N}$, with $N \in \mathbb{N}_{*}$ and let $\alpha$ and $\beta$ be two real numbers, with $0<\alpha \leq \beta$. We denote by $\mathcal{M}(\alpha, \beta, \Omega)$ the set of measurable functions $M: \Omega \longrightarrow \mathcal{L}\left(\mathbb{R}^{N}, \mathbb{R}^{N}\right)$ such that, for a.e. $x \in \Omega$ and for all $(\xi, \chi) \in\left(\mathbb{R}^{N}\right)^{2}, \alpha|\xi|^{2} \leq M(x) \xi \cdot \xi \leq \beta|\xi|^{2}$, and $M(x) \xi \cdot \chi=\xi \cdot M(x) \chi$. In the particular case where there exists a function $\mu \in L^{\infty}(\Omega)$ such that, for a.e. $x \in \Omega, M(x)=\mu(x) I_{N}$, where $I_{N}$ denotes the identity application from $\mathbb{R}^{N}$ to $\mathbb{R}^{N}$, we then denote $M=\mu$. In this case, we say that $M$ represents an isotropic field; otherwise, we say that the field $M$ is anisotropic.

For a given source term $b \in H^{-1}(\Omega)$ and a given $M \in \mathcal{M}(\alpha, \beta, \Omega)$, we denote by $\mathcal{F}(b, M)$ the unique solution $\bar{u}$ of

$$
\bar{u} \in H_{0}^{1}(\Omega) \text { and } \int_{\Omega} M(x) \nabla \bar{u}(x) \cdot \nabla \bar{v}(x) d x=b(\bar{v}), \forall \bar{v} \in H_{0}^{1}(\Omega) .
$$

\subsection{The H-convergence theorem}

The following result, given in [17] (in which it was called G-convergence, in reference to some de Giorgi's works), has been extended in [19] to some more general configurations.

Theorem 1 (H-convergence) Let $\Omega$ be an open bounded subset of $\mathbb{R}^{N}$, with $N \in \mathbb{N}_{*}$. Let two real numbers $\alpha$ and $\beta$ be such that $0<\alpha \leq \beta$. Let $\left(M_{n}\right)_{n \in \mathbb{N}}$ be a sequence of elements of $M_{n} \in \mathcal{M}(\alpha, \beta, \Omega)$. Then there exists a subsequence of $\left(M_{n}\right)_{n \in \mathbb{N}}$, again denoted $\left(M_{n}\right)_{n \in \mathbb{N}}$, and a function $M \in \mathcal{M}(\alpha, \beta, \Omega)$ such that:

- for all $b \in H^{-1}(\Omega), \mathcal{F}\left(b, M_{n}\right)$ weakly converges to $\mathcal{F}(b, M)$ in $H_{0}^{1}(\Omega)$ as $n \longrightarrow \infty$,

- for all $b \in H^{-1}(\Omega), M_{n} \nabla \mathcal{F}\left(b, M_{n}\right)$ weakly converges to $M \nabla \mathcal{F}(b, M)$ in $\left(L^{2}(\Omega)\right)^{N}$ as $n \longrightarrow \infty$.

We then say that the sequence $\left(M_{n}\right)_{n \in \mathbb{N}} H$-converges to $M$, called the H-limit of the sequence.

We now give some examples of H-convergence results.

\subsection{The one-dimensional case}

In the case $N=1$, let us suppose that $\Omega=(0,1)$. The sequence $\left(M_{n}\right)_{n \in \mathbb{N}}$ such that for all $n \in \mathbb{N}$, $M_{n} \in \mathcal{M}(\alpha, \beta, \Omega)$ is then a sequence of functions belonging to $L^{\infty}(\Omega)$ and $1 / M_{n}(x) \in[1 / \beta, 1 / \alpha]$ for a.e. $x \in \Omega$. For a given $f \in L^{2}(\Omega)$, we denote by $\hat{f}$ the continuous function defined, for all $x \in(0,1)$, by $\hat{f}(x)=\int_{(0, x)} f(s) d s$. We then have, for all $x \in \Omega$,

$$
\mathcal{F}\left(f, M_{n}\right)(x)=\frac{\int_{(0, x)}\left(1 / M_{n}(t)\right) d t}{\int_{(0,1)}\left(1 / M_{n}(t)\right) d t} \int_{(0,1)} \frac{\hat{f}(t)}{M_{n}(t)} d t-\int_{(0, x)} \frac{\hat{f}(t)}{M_{n}(t)} d t .
$$


Up to a subsequence, we can suppose that the sequence $\left(1 / M_{n}\right)_{n \in \mathbb{N}}$ converges to a function $1 / M$ for the weak $\star$ topology of $L^{\infty}(\Omega)$. We then get that, for all $x \in \Omega$,

$$
\lim _{n \longrightarrow \infty} \mathcal{F}\left(f, M_{n}\right)(x)=\frac{\int_{(0, x)}(1 / M(t)) d t}{\int_{(0,1)}(1 / M(t)) d t} \int_{(0,1)} \frac{\hat{f}(t)}{M(t)} d t-\int_{(0, x)} \frac{\hat{f}(t)}{M(t)} d t,
$$

which proves that $M$ is the H-limit of this subsequence. Unfortunately, such a relation between the limit for the weak $\star$ topology of $L^{\infty}(\Omega)$ and the H-limit cannot be obtained in the general case $N>1$.

\subsection{Two-dimensional examples}

Let $\mu_{r}>0$ and $\mu_{b}>0$ be two real values respectively defining the permeability of two materials, respectively called "red" and "black". We first define the so-called checkerboard problem, setting $M_{1}$ : $\mathbb{R}^{2} \longrightarrow \mathbb{R}$ by $\left(x_{1}, x_{2}\right) \rightarrow \mu_{r}$ if $\operatorname{Int}\left(x_{1}\right)+\operatorname{Int}\left(x_{2}\right) \in 2 \mathbb{Z}$ (denoting for all $z \in \mathbb{R}$ by $\operatorname{Int}(z)$ the largest relative integer value lower than $z$ ), else $\left(x_{1}, x_{2}\right) \rightarrow \mu_{b}$ (for example, $\operatorname{Int}(0.5)+\operatorname{Int}(0.5)=0$ and $M_{1}(0.5,0.5)=\mu_{r}$, $\operatorname{Int}(1.5)+\operatorname{Int}(-1.5)=1-2=-1$ and $M_{1}(1.5,-1.5)=\mu_{b}$ : see Figure 1). Then we define, for all $n \in \mathbb{N}_{*}$, $M_{n}: \mathbb{R}^{2} \longrightarrow \mathbb{R}$ by $M_{n}\left(x_{1}, x_{2}\right)=M_{1}\left(n x_{1}, n x_{2}\right)$. It can then be shown that, in all open domain $\Omega$ of $\mathbb{R}^{2}$, the sequence $\left(M_{n}\right)_{n \in \mathbb{N}} \mathrm{H}$-converges to the constant function $\left(x_{1}, x_{2}\right) \rightarrow \sqrt{\mu_{r} \mu_{b}}$. In this case, the H-limit of a sequence of isotropic heterogeneous fields is an isotropic homogeneous field.

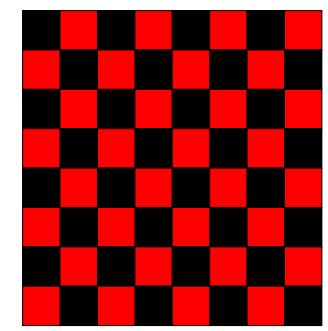

Figure 1: The checkerboard case

Another example involving two materials is the multilayer case, obtained with defining $M_{1}: \mathbb{R}^{2} \longrightarrow \mathbb{R}$ by $\left(x_{1}, x_{2}\right) \rightarrow \mu_{r}$ if $\operatorname{Int}\left(x_{1}\right) \in 2 \mathbb{Z}$, else $\left(x_{1}, x_{2}\right) \rightarrow \mu_{b}$ (for example, $\operatorname{Int}(0.5)=0$ and $M_{1}(0.5,10)=\mu_{r}$, $\operatorname{Int}(1.5)=1$ and $M_{1}(1.5,-4)=\mu_{b}$ : see Figure 2).

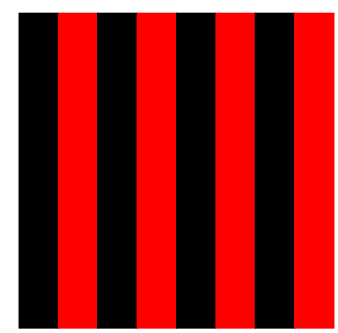

Figure 2: The multilayer case

We again define the sequence $\left(M_{n}\right)_{n \in \mathbb{N}}$, by $M_{n}: \mathbb{R}^{2} \longrightarrow \mathbb{R},\left(x_{1}, x_{2}\right) \mapsto M_{1}\left(n x_{1}, n x_{2}\right)$, for all $n \in \mathbb{N}_{*}$. Then it can be proved that the sequence $\left(M_{n}\right)_{n \in \mathbb{N}}$ H-converges, in all open domain $\Omega$ of $\mathbb{R}^{2}$, to the constant field, the value of which is the linear function defined by $(1,0) \rightarrow\left(\frac{2 \mu_{r} \mu_{b}}{\mu_{r}+\mu_{b}}, 0\right)$ and $(0,1) \rightarrow$ $\left(0, \frac{\mu_{r}+\mu_{b}}{2}\right)$. We can remark that $\frac{2 \mu_{r} \mu_{b}}{\mu_{r}+\mu_{b}}$ is the harmonic average of $\mu_{r}$ and $\mu_{b}$, that is the invert of the average value of the inverts of $\mu_{r}$ and $\mu_{b}$ (this is exactly the value obtained by H-convergence in the 
one-dimensional case), whereas $\frac{\mu_{r}+\mu_{b}}{2}$ is the arithmetic average of $\mu_{r}$ and $\mu_{b}$. In this two-dimensional case, the H-limit of a sequence of isotropic heterogeneous fields is an anisotropic homogeneous field.

Note that in the two above examples, the limit of $\left(M_{n}\right)_{n \in \mathbb{N}}$ for the weak $\star$ topology of $L^{\infty}(\Omega)$ is the constant function $\left(x_{1}, x_{2}\right) \rightarrow \frac{\mu_{r}+\mu_{b}}{2}$. Using the notion of nonlinear weak $\star$ convergence (see [9]), the limit of $\left(M_{n}\right)_{n \in \mathbb{N}}$ in terms of Young's measure is the constant field of probability measure $\frac{1}{2} \delta_{\mu_{r}}+\frac{1}{2} \delta_{\mu_{b}}$, equivalently given by the function $\mu \in L^{\infty}(\Omega \times(0,1))$ such that, for a.e. $x \in \Omega$ and $s \in\left(0, \frac{1}{2}\right), \mu(x, s)=\mu_{r}$ and for a.e. $x \in \Omega$ and $s \in\left(\frac{1}{2}, 1\right), \mu(x, s)=\mu_{b}$. Thus we see that the notion of nonlinear weak $\star$ convergence does not account for the spatial structure of the heterogenity, and justifies the attempts of finding some more suitable generalized limit (see for example [20]).

\section{Finite Volume meshes and schemes}

\subsection{Admissible meshes}

We first introduce the notion of admissible discretization [9] which is useful to define a finite volume scheme.

Definition 1 (Admissible discretization) Let $\Omega$ be an open bounded polygonal subset of $\mathbb{R}^{N}$, with $N \in \mathbb{N}_{*}$ (in general, we have $N=2$ or $N=3$ ). We denote $\partial \Omega=\bar{\Omega} \backslash \Omega$. An admissible finite volume discretization of $\Omega$, denoted by $\mathcal{D}$, is given by $\mathcal{D}=(\mathcal{T}, \mathcal{E}, \mathcal{P})$, where:

- $\mathcal{T}$ is a finite family of non empty open polygonal convex disjoint subsets of $\Omega$ (the "control volumes") such that $\bar{\Omega}=\cup_{K \in \mathcal{T}} \bar{K}$. We then denote, for all $K \in \mathcal{T}$, by $\partial K=\bar{K} \backslash K$ the boundary of $K$ and $m_{K}>0$ the $N$-dimensional Lebesgue measure of $K$ (it is the area of $K$ in the two-dimensional case and the volume in the three-dimensional case).

- $\mathcal{E}$ is a finite family of disjoint subsets of $\bar{\Omega}$ (the "edges" of the mesh), such that, for all $\sigma \in \mathcal{E}$, there exists a hyperplane $E$ of $\mathbb{R}^{N}$ and $K \in \mathcal{T}$ with $\bar{\sigma}=\partial K \cap E$ and $\sigma$ is a non empty open subset of $E$. We then denote $m_{\sigma}>0$ the $(N-1)$-dimensional measure of $\sigma$. We assume that, for all $K \in \mathcal{T}$, there exists a subset $\mathcal{E}_{K}$ of $\mathcal{E}$ such that $\partial K=\cup_{\sigma \in \mathcal{E}_{K}} \bar{\sigma}$. It then results from the previous hypotheses that, for all $\sigma \in \mathcal{E}$, either $\sigma \subset \partial \Omega$ or there exists $(K, L) \in \mathcal{T}^{2}$ with $K \neq L$ such that $\bar{K} \cap \bar{L}=\bar{\sigma}$; we denote in the latter case $\sigma=K \mid L$.

- $\mathcal{P}$ is a family of points of $\Omega$ indexed by $\mathcal{T}$, denoted by $\mathcal{P}=\left(x_{K}\right)_{K \in \mathcal{T}}$. This family is such that, for all $K \in \mathcal{T}, x_{K} \in K$. For all $\sigma \in \mathcal{E}$ such that there exists $(K, L) \in \mathcal{T}^{2}$ with $\sigma=K \mid L$, it is assumed that the straight line $\left(x_{K}, x_{L}\right)$ going through $x_{K}$ and $x_{L}$ is orthogonal to $K \mid L$. For all $K \in \mathcal{T}$ and all $\sigma \in \mathcal{E}_{K}$, let $y_{\sigma}$ be the orthogonal projection of $x_{K}$ on $\sigma$. We suppose that $y_{\sigma} \in \sigma$.

The following notations are used. The size of the discretization is defined by:

$$
\operatorname{size}(\mathcal{D})=\sup \{\operatorname{diam}(K), K \in \mathcal{T}\} .
$$

For all $K \in \mathcal{T}$ and $\sigma \in \mathcal{E}_{K}$, we denote by $\mathbf{n}_{K, \sigma}$ the unit vector normal to $\sigma$ outward to $K$. We define a subset of $K$ associated with the edge $\sigma$ by

$$
D_{K, \sigma}=\left\{t x_{K}+(1-t) y, t \in(0,1), y \in \sigma\right\}
$$

(the letter " $D$ " stands for "diamond") and denote by $d_{K, \sigma}$ the euclidean distance between $x_{K}$ and $\sigma$. We then define

$$
\tau_{K, \sigma}=\frac{m_{\sigma}}{d_{K, \sigma}} .
$$

The set of interior (resp. boundary) edges is denoted by $\mathcal{E}_{\text {int }}$ (resp. $\mathcal{E}_{\text {ext }}$ ), that is $\mathcal{E}_{\text {int }}=\{\sigma \in \mathcal{E} ; \sigma \not \subset \partial \Omega\}$ (resp. $\mathcal{E}_{\text {ext }}=\{\sigma \in \mathcal{E} ; \sigma \subset \partial \Omega\}$ ). 


\subsection{Discrete functional properties}

Definition 2 Let $\Omega$ be an open bounded polygonal subset of $\mathbb{R}^{N}$, with $N \in \mathbb{N}_{*}$. Let $\mathcal{D}=(\mathcal{T}, \mathcal{E}, \mathcal{P})$ be an admissible finite volume discretization of $\Omega$ in the sense of Definition 1. We denote by $H_{\mathcal{D}}(\Omega) \subset L^{2}(\Omega)$ the space of functions which admit a constant value in each $K \in \mathcal{T}$. For all $u \in H_{\mathcal{D}}(\Omega)$ and for all $K \in \mathcal{T}$, we denote by $u_{K}$ the constant value of $u$ in $K$ and we define $\left(u_{\sigma}\right)_{\sigma \in \mathcal{E}}$ by

$$
u_{\sigma}=0, \forall \sigma \in \mathcal{E}_{\text {ext }}
$$

and

$$
\tau_{K, \sigma}\left(u_{\sigma}-u_{K}\right)+\tau_{L, \sigma}\left(u_{\sigma}-u_{L}\right)=0, \forall \sigma \in \mathcal{E}_{\text {int }}, \sigma=K \mid L .
$$

We now give a definition of an approximate gradient of the functions of $H_{\mathcal{D}}(\Omega)$. We define a function, denoted $\mathbf{G}_{\mathcal{D}}: H_{\mathcal{D}}(\Omega) \longrightarrow\left(L^{2}(\Omega)\right)^{N}, u \longrightarrow \mathbf{G}_{\mathcal{D}} u$ with

$$
\mathbf{G}_{\mathcal{D}} u(x)=\frac{N}{d_{K, \sigma}}\left(u_{\sigma}-u_{K}\right) \mathbf{n}_{K, \sigma}, \text { for a.e. } x \in D_{K, \sigma}, \forall K \in \mathcal{T}, \forall \sigma \in \mathcal{E}_{K} .
$$

Let two real numbers $\alpha$ and $\beta$ be such that $0<\alpha \leq \beta$. We denote by $\mathcal{M}_{\mathcal{D}}(\alpha, \beta) \subset L^{\infty}(\Omega)$ the set of functions $\mu$ such that for all $\sigma \in \mathcal{E}$, there exists a constant value, denoted $\mu_{\sigma} \in[\alpha, \beta]$, such that

$$
\mu(x)=\mu_{\sigma}, \forall x \in D_{K, \sigma} \text { where } K \text { is such that } \sigma \in \mathcal{E}_{K} .
$$

The function which takes the constant value 1 on $\Omega$ is denoted by 1 . For $(u, v) \in\left(H_{\mathcal{D}}(\Omega)\right)^{2}$ and $\varphi \in C^{0}(\bar{\Omega})$, we denote by

$$
[u, v]_{\mathcal{D}, \mu, \varphi}=\sum_{K \in \mathcal{T}} \varphi\left(x_{K}\right) \sum_{\sigma \in \mathcal{E}_{K}} \mu_{\sigma} \tau_{K, \sigma}\left(u_{\sigma}-u_{K}\right)\left(v_{\sigma}-v_{K}\right) .
$$

We define the following norm in $H_{\mathcal{D}}(\Omega)$ (see Lemma 1) by

$$
|u|_{\mathcal{D}}=\left([u, u]_{\mathcal{D}, 1,1}\right)^{1 / 2} .
$$

Remark 1 For all edge $\sigma$ such that $\sigma=K \mid L$, the function $\mathbf{G}_{\mathcal{D}} u$ is constant on $D_{K, \sigma} \cup D_{L, \sigma}$.

We have the following properties.

Lemma 1 (Discrete Poincaré inequality) Let $\Omega$ be an open bounded polygonal subset of $\mathbb{R}^{N}$, with $N \in \mathbb{N}_{*}$. Let $\mathcal{D}=(\mathcal{T}, \mathcal{E}, \mathcal{P})$ be an admissible finite volume discretization of $\Omega$ in the sense of Definition 1. Then for all $u \in H_{\mathcal{D}}(\Omega)$ (cf Definition 2), one has

$$
\|u\|_{L^{2}(\Omega)} \leq \operatorname{diam}(\Omega)|u|_{\mathcal{D}}
$$

The proof of Lemma 1 is given in [9].

Lemma 2 (Relative compactness in $L^{2}(\Omega)$ ) Let $\Omega$ be an open bounded polygonal subset of $\mathbb{R}^{N}$, with $N \in \mathbb{N}_{*}$. We consider a sequence $\left(\mathcal{D}_{n}, u_{n}\right)_{n \in \mathbb{N}}$ such that, for all $n \in \mathbb{N}, \mathcal{D}_{n}$ is an admissible finite volume discretization of $\Omega$ in the sense of Definition 1 and $u_{n} \in H_{\mathcal{D}_{n}}(\Omega)$ (cf Definition 2). Let us assume that

$$
\lim _{n \longrightarrow \infty} \operatorname{size}\left(\mathcal{D}_{n}\right)=0
$$

and that there exists $C>0$ such that, for all $n \in \mathbb{N},\left|u_{n}\right|_{\mathcal{D}_{n}} \leq C$. 
Then there exists a subsequence of $\left(\mathcal{D}_{n}, u_{n}\right)_{n \in \mathbb{N}}$, again denoted $\left(\mathcal{D}_{n}, u_{n}\right)_{n \in \mathbb{N}}$, and $\bar{u} \in H_{0}^{1}(\Omega)$ such that $u_{n}$ tends to $\bar{u}$ in $L^{2}(\Omega)$ as $n \longrightarrow \infty, \mathbf{G}_{\mathcal{D}_{n}} u_{n}$ weakly tends to $\nabla \bar{u}$ in $\left(L^{2}(\Omega)\right)^{N}$ as $n \longrightarrow \infty$ and

$$
\int_{\Omega} \varphi(x)(\nabla \bar{u}(x))^{2} d x \leq \liminf _{n \longrightarrow \infty}\left[u_{n}, u_{n}\right]_{\mathcal{D}_{n}, 1, \varphi}, \quad \forall \varphi \in C^{0}\left(\bar{\Omega}, \mathbb{R}_{+}\right) .
$$

Proof The proof of the existence of a subsequence of $\left(\mathcal{D}_{n}, u_{n}\right)_{n \in \mathbb{N}}$, again denoted $\left(\mathcal{D}_{n}, u_{n}\right)_{n \in \mathbb{N}}$, and $\bar{u} \in H_{0}^{1}(\Omega)$ such that $u_{n}$ tends to $\bar{u}$ in $L^{2}(\Omega)$ as $n \longrightarrow \infty$ is given in [9]. The proof of (9) is given in [10]. Therefore, we only have to prove that, up to a subsequence, $\mathbf{G}_{\mathcal{D}_{n}} u_{n}$ weakly tends to $\nabla \bar{u}$ in $\left(L^{2}(\Omega)\right)^{N}$ as $n \longrightarrow \infty$. Since for all $K \in \mathcal{T}$ and $\sigma \in \mathcal{E}_{K}$ the measure of $D_{K, \sigma}$ is equal to $m_{\sigma} d_{K, \sigma} / N$, we have

$$
\left(\left\|\mathbf{G}_{\mathcal{D}_{n}} u_{n}\right\|_{\left(L^{2}(\Omega)\right)^{N}}\right)^{2}=N\left(\left|u_{n}\right|_{\mathcal{D}_{n}}\right)^{2} \leq N C^{2} .
$$

Thus there exists a subsequence of $\left(\mathcal{D}_{n}, u_{n}\right)_{n \in \mathbb{N}}$, again denoted $\left(\mathcal{D}_{n}, u_{n}\right)_{n \in \mathbb{N}}$ and $\overline{\mathbf{g}} \in\left(L^{2}(\Omega)\right)^{N}$ such that $\mathbf{G}_{\mathcal{D}_{n}} u_{n}$ converges weakly to $\overline{\mathbf{g}}$ in $\left(L^{2}(\Omega)\right)^{N}$ as $n \longrightarrow \infty$. It now remains to prove that $\overline{\mathbf{g}}=\nabla \bar{u}$. Let $\varphi \in\left(C_{c}^{\infty}(\Omega)\right)^{N}$. Let $G_{0}$ be defined by:

$$
G_{0}=\int_{\Omega} \nabla \bar{u}(x) \cdot \varphi(x) d x=-\int_{\Omega} \bar{u}(x) \operatorname{div} \varphi(x) d x .
$$

For a given $n \in \mathbb{N}$, we consider the expression

$$
G_{0, n}=-\int_{\Omega} u_{n} \operatorname{div} \varphi(x) d x .
$$

We then have $\lim _{n \longrightarrow \infty} G_{0, n}=G_{0}$. Since we have (omitting indexes $n$ in discrete terms)

$$
G_{0, n}=-\sum_{K \in \mathcal{T}} u_{K} \int_{K} \operatorname{div} \varphi(x) d x=\sum_{K \in \mathcal{T}} \sum_{\sigma \in \mathcal{E}_{K}}\left(u_{\sigma}-u_{K}\right) \int_{\sigma} \varphi(x) \cdot \mathbf{n}_{K, \sigma} d \gamma(x),
$$

(in which $d \gamma(x)$ is the $N$-1-dimensional measure) we get

$$
G_{0, n}=\sum_{K \in \mathcal{T}} \sum_{\sigma \in \mathcal{E}_{K}} \frac{N}{d_{K, \sigma}}\left(u_{\sigma}-u_{K}\right) \int_{D_{K, \sigma}} \varphi_{\sigma} \cdot \mathbf{n}_{K, \sigma} d x,
$$

in which we denote

$$
\varphi_{\sigma}=\frac{1}{m_{\sigma}} \int_{\sigma} \varphi(x) d \gamma(x), \forall \sigma \in \mathcal{E}
$$

If we now set

$$
G_{1, n}=\int_{\Omega} \mathbf{G}_{\mathcal{D}_{n}} u_{n}(x) \cdot \varphi(x) d x
$$

we have on the one hand $\lim _{n \longrightarrow \infty} G_{1, n}=\int_{\Omega} \overline{\mathbf{g}}(x) \cdot \varphi(x) d x$ and on the other hand

$$
G_{1, n}=\sum_{K \in \mathcal{T}} \sum_{\sigma \in \mathcal{E}_{K}} \frac{N}{d_{K, \sigma}}\left(u_{\sigma}-u_{K}\right) \int_{D_{K, \sigma}} \varphi(x) \cdot \mathbf{n}_{K, \sigma} d x .
$$

Therefore, denoting $C_{0, \varphi}>0$ a value such that $|\varphi(x)-\varphi(y)| \leq C_{0, \varphi}|x-y|$, we have

$$
\left|G_{1, n}-G_{0, n}\right| \leq C_{0, \varphi} \operatorname{size}\left(\mathcal{D}_{n}\right) \sum_{K \in \mathcal{T}} \sum_{\sigma \in \mathcal{E}_{K}} m_{\sigma}\left|u_{\sigma}-u_{K}\right|,
$$

and thanks to the Cauchy-Schwarz inequality, 


$$
\begin{aligned}
\left(G_{1, n}-G_{0, n}\right)^{2} & \leq C_{0, \varphi}^{2} \operatorname{size}\left(\mathcal{D}_{n}\right)^{2} N m_{\Omega} \sum_{K \in \mathcal{T}} \sum_{\sigma \in \mathcal{E}_{K}} \tau_{K, \sigma}\left(u_{\sigma}-u_{K}\right)^{2} \\
& \leq C_{0, \varphi}^{2} \operatorname{size}\left(\mathcal{D}_{n}\right)^{2} N m_{\Omega} C
\end{aligned}
$$

where $m_{\Omega}$ denotes the measure of $\Omega$ which verifies $N m_{\Omega}=\sum_{K \in \mathcal{T}} \sum_{\sigma \in \mathcal{E}_{K}} m_{\sigma} d_{K, \sigma}$.

This proves that $\lim _{n \longrightarrow \infty} G_{0, n}=\lim _{n \longrightarrow \infty} G_{1, n}$, and therefore

$$
\int_{\Omega} \overline{\mathbf{g}}(x) \cdot \varphi(x) d x=\int_{\Omega} \nabla \bar{u}(x) \cdot \varphi(x) d x .
$$

Since the above equation is true for all $\varphi \in\left(C_{c}^{\infty}(\Omega)\right)^{N}$, we then deduce that $\overline{\mathbf{g}}(x)=\nabla \bar{u}(x)$, for a.e. $x \in \Omega$. Thanks to the uniqueness of this limit, this proves that all the sequence $\left(\mathcal{D}_{n}, u_{n}\right)_{n \in \mathbb{N}}$ such that $u_{n}$ tends to $\bar{u}$ in $L^{2}(\Omega)$ as $n \longrightarrow \infty$ verifies that $\mathbf{G}_{\mathcal{D}_{n}} u_{n}$ weakly tends to $\nabla \bar{u}$ in $\left(L^{2}(\Omega)\right)^{N}$ as $n \longrightarrow \infty$.

Remark 2 In the preceding proof, the convergence of $\mathbf{G}_{\mathcal{D}_{n}} u_{n}$, as $n \longrightarrow \infty$, cannot be in $\left(L^{2}(\Omega)\right)^{N}$, except if it converges to 0 , since we get $\liminf _{n}\left\|\mathbf{G}_{\mathcal{D}_{n}} u_{n}\right\|_{\left(L^{2}(\Omega)\right)^{N}} \geq \sqrt{N}\|\nabla \bar{u}\|_{\left(L^{2}(\Omega)\right)^{N}}$ from (10) and (9) with $\varphi=1$ (see also Remark 7 for a more general case).

\subsection{Finite volume scheme}

We now give a finite volume scheme for a Dirichlet problem on $\Omega$. Let $\mathcal{D}=(\mathcal{T}, \mathcal{E}, \mathcal{P})$ be an admissible discretization of $\Omega$ in the sense of Definition 1. Let two real numbers $\alpha$ and $\beta$ be such that $0<\alpha \leq \beta$ and let $\mu \in \mathcal{M}_{\mathcal{D}}(\alpha, \beta)$. For a given $f \in L^{2}(\Omega)$, let $u \in H_{\mathcal{D}}(\Omega)$ (cf Definition 2) be such that

$$
-\sum_{\sigma \in \mathcal{E}_{K}} \mu_{\sigma} \tau_{K, \sigma}\left(u_{\sigma}-u_{K}\right)=\int_{K} f(x) d x, \forall K \in \mathcal{T}
$$

(the existence and uniqueness of $u \in H_{\mathcal{D}}(\Omega)$ solution of (11) results from the inequality $\mu_{\sigma} \geq \alpha$ for all $\sigma \in \mathcal{E}$ and from (8), see [9]). Since, for all $v \in H_{\mathcal{D}}(\Omega), \sum_{K \in \mathcal{T}} \sum_{\sigma \in \mathcal{E}_{K}} \mu_{\sigma} \tau_{K, \sigma}\left(u_{\sigma}-u_{K}\right) v_{\sigma}=0$ thanks to (4)-(5), (11) is equivalent to

$$
u \in H_{\mathcal{D}}(\Omega) \text { and }[u, v]_{\mathcal{D}, \mu, 1}=\int_{\Omega} f(x) v(x) d x, \forall v \in H_{\mathcal{D}}(\Omega) .
$$

Using the results of Lemma 2 for the points concerning the approximate gradient, we then have the following results, given in [9].

Lemma 3 (Finite volume method) Let $\Omega$ be an open bounded polygonal subset of $\mathbb{R}^{N}$, with $N \in \mathbb{N}_{*}$. Let $\mathcal{D}=(\mathcal{T}, \mathcal{E}, \mathcal{P})$ be an admissible finite volume discretization of $\Omega$ in the sense of Definition 1. Let two real numbers $\alpha$ and $\beta$ be such that $0<\alpha \leq \beta$ and let $\mu \in \mathcal{M}_{\mathcal{D}}(\alpha, \beta)$. Let $f \in L^{2}(\Omega)$.

Then there exists one and only one $u \in H_{\mathcal{D}}(\Omega)$ (cf Definition 2) given by (11). We then denote $u=$ $F_{\mathcal{D}}(f, \mu)$. Moreover,

$$
\alpha|u|_{\mathcal{D}} \leq \operatorname{diam}(\Omega)\|f\|_{L^{2}(\Omega)} .
$$

In the case $\mu=1$, we have the following convergence results: $F_{\mathcal{D}}(f, 1)$ converges to $\mathcal{F}(f, 1)$ in $L^{2}(\Omega)$ as $\operatorname{size}(\mathcal{D}) \longrightarrow 0, \mathbf{G}_{\mathcal{D}} F_{\mathcal{D}}(f, 1)$ weakly converges to $\nabla \mathcal{F}(f, 1)$ as $\operatorname{size}(\mathcal{D}) \longrightarrow 0$ in $\left(L^{2}(\Omega)\right)^{N}$, and

$$
\int_{\Omega} \varphi(x)(\nabla \mathcal{F}(f, 1)(x))^{2} d x=\lim _{\operatorname{size}(\mathcal{D}) \longrightarrow 0}\left[F_{\mathcal{D}}(f, 1), F_{\mathcal{D}}(f, 1)\right]_{\mathcal{D}, 1, \varphi}, \quad \forall \varphi \in C^{0}(\bar{\Omega}) .
$$




\section{Adaptation of $\mathrm{H}$-convergence to numerical schemes}

\subsection{The Hd-convergence theorem and relations with $\mathrm{H}$-convergence}

The following theorem (proved in Sections 4.2 and 4.3 below) expresses a discrete version of Theorem 1 .

Theorem 2 (Hd-convergence) Let $\Omega$ be an open bounded polygonal subset of $\mathbb{R}^{N}$, with $N \in \mathbb{N}_{*}$. Let two real numbers $\alpha$ and $\beta$ be such that $0<\alpha \leq \beta$. Let $\left(\mathcal{D}_{n}, \mu_{n}\right)_{n \in \mathbb{N}}$ be a sequence such that, for all $n \in \mathbb{N}, \mathcal{D}_{n}$ is an admissible discretization of $\Omega$ in the sense of Definition 1 , and $\mu_{n} \in \mathcal{M}_{\mathcal{D}_{n}}(\alpha, \beta)$. We assume that $\lim _{n \rightarrow \infty} \operatorname{size}\left(\mathcal{D}_{n}\right)=0$.

Then there exist a subsequence of $\left(\mathcal{D}_{n}, \mu_{n}\right)_{n \in \mathbb{N}}$, again denoted $\left(\mathcal{D}_{n}, \mu_{n}\right)_{n \in \mathbb{N}}$, and a unique measurable function $M \in \mathcal{M}(\alpha, \beta, \Omega)$ (this set is defined in Section 2) such that:

- for all $f \in L^{2}(\Omega), F_{\mathcal{D}_{n}}\left(f, \mu_{n}\right)$ converges to $\mathcal{F}(f, M)$ in $L^{2}(\Omega)$ as $n \longrightarrow \infty$ and $\mathbf{G}_{\mathcal{D}_{n}} F_{\mathcal{D}_{n}}\left(f, \mu_{n}\right)$ weakly converges to $\nabla \mathcal{F}(f, M)$ in $\left(L^{2}(\Omega)\right)^{N}$ as $n \longrightarrow \infty$ (the functions $F_{\mathcal{D}}(f, \mu)$, denoting the discrete solution of a finite volume scheme for an elliptic problem with the homogeneous Dirichlet boundary condition, the right hand side $f$ and a discrete diffusion field $\mu$, and $\mathbf{G}_{\mathcal{D}} F_{\mathcal{D}}(f, \mu)$, denoting a discrete gradient of this numerical solution, are defined in Section 3 and the function $\mathcal{F}(f, M)$, denoting the solution of an elliptic problem with the homogeneous Dirichlet boundary condition, the right hand side $f$ and a diffusion matrix field $M$, is defined in Section 2),

- for all $f \in L^{2}(\Omega), \mu_{n} \mathbf{G}_{\mathcal{D}_{n}} F_{\mathcal{D}_{n}}\left(f, \mu_{n}\right)$ weakly converges to $M \nabla \mathcal{F}(f, M)$ in $\left(L^{2}(\Omega)\right)^{N}$ as $n \longrightarrow \infty$.

We then say that the sequence $\left(\mathcal{D}_{n}, \mu_{n}\right)_{n \in \mathbb{N}} H d$-converges to $M$, called the Hd-limit of the sequence.

Some comments can be done on the relation between Hd-convergence and H-convergence. Let us first study the one-dimensional case. We take again the case and the notations of Section 2.3 . Let $\Omega=(0,1)$, $\alpha$ and $\beta$ be such that $0<\alpha \leq \beta$. In order to define an admissible discretization of $\Omega$, let $p \in \mathbb{N}_{*}$ and let $\left(y_{k}\right)_{k=0, \ldots, p}$ and $\left(x_{k}\right)_{k=1, \ldots, p}$ be real values such that

$$
y_{0}=0<x_{1}<y_{1}<x_{2} \ldots<y_{k-1}<x_{k}<y_{k} \ldots<y_{p-1}<x_{p}<y_{p}=1 \text {. }
$$

Then the discretization $\mathcal{D}=(\mathcal{T}, \mathcal{E}, \mathcal{P})$ defined by $\mathcal{T}=\left\{\left(y_{k-1}, y_{k}\right), k=1, \ldots, p\right\}, \mathcal{E}=\left\{\left\{y_{k}\right\}, k=0, \ldots, p\right\}$ and $\mathcal{P}=\left\{x_{k}, k=1, \ldots, p\right\}$ is an admissible discretization of $\Omega$ in the sense of Definition 1 . Let $f \in L^{2}(\Omega)$ and $\mu \in \mathcal{M}_{\mathcal{D}}(\alpha, \beta)$ be given (recall that the function $\mu$ takes constant values in $\left(0, x_{1}\right), \ldots,\left(x_{k}, x_{k+1}\right), \ldots$, $\left.\left(x_{p}, 1\right)\right)$. We again define the function $\hat{f}$ by $\hat{f}(x)=\int_{(0, x)} f(t) d t$ for all $x \in \Omega$, and we introduce the function $\hat{f}_{\mathcal{D}}$ defined, by $\hat{f}_{\mathcal{D}}(x)=0=\hat{f}\left(y_{0}\right)$ for all $x \in\left(0, x_{1}\right)$, by $\hat{f}_{\mathcal{D}}(x)=\hat{f}\left(y_{k}\right)$ for all $x \in\left(x_{k}, x_{k+1}\right)$, and by $\hat{f}_{\mathcal{D}}(x)=\hat{f}(1)$ for all $x \in\left(x_{p}, 1\right)$. Some calculations show that the solution of the finite volume scheme (11) is defined by

$$
\begin{aligned}
& F_{\mathcal{D}}(f, \mu)(x)=\frac{\int_{\left(0, x_{k}\right)}(1 / \mu(t)) d t}{\int_{(0,1)}(1 / \mu(t)) d t} \int_{(0,1)} \frac{\hat{f}_{\mathcal{D}}(t)}{\mu(t)} d t-\int_{\left(0, x_{k}\right)} \frac{\hat{f}_{\mathcal{D}}(t)}{\mu(t)} d t, \\
& \forall x \in\left(y_{k-1}, y_{k}\right), \quad \forall k=1, \ldots, p .
\end{aligned}
$$

Let $\left(\mathcal{D}_{n}, \mu_{n}\right)_{n \in \mathbb{N}}$ be a sequence such that, for all $n \in \mathbb{N}, \mathcal{D}_{n}$ is an admissible discretization of $\Omega$ in the sense of Definition 1 , and $\mu_{n} \in \mathcal{M}_{\mathcal{D}_{n}}(\alpha, \beta)$. We assume that $\lim _{n \rightarrow \infty} \operatorname{size}\left(\mathcal{D}_{n}\right)=0$. Up to a subsequence, we can suppose that the sequence $\left(1 / \mu_{n}\right)_{n \in \mathbb{N}}$ converges to a function $1 / M$ for the weak $\star$ topology of $L^{\infty}(\Omega)$. Since the sequence $\left(\hat{f}_{\mathcal{D}_{n}}\right)_{n \in \mathbb{N}}$ strongly converges to the continuous function $\hat{f}$ as $n \longrightarrow \infty$, we get, using (15) in which we let $\mathcal{D}=\mathcal{D}_{n}$ and $\mu=\mu_{n}$, that the limit of the sequence $\left(F_{\mathcal{D}_{n}}\left(f, \mu_{n}\right)\right)_{n \in \mathbb{N}}$ is exactly the function $\mathcal{F}(f, M)$ defined, for all $x \in \Omega$, by

$$
\mathcal{F}(f, M)(x)=\frac{\int_{(0, x)}(1 / M(t)) d t}{\int_{(0,1)}(1 / M(t)) d t} \int_{(0,1)} \frac{\hat{f}(t)}{M(t)} d t-\int_{(0, x)} \frac{\hat{f}(t)}{M(t)} d t .
$$


This proves that the Hd-limit of $\left(\mathcal{D}_{n}, \mu_{n}\right)_{n \in \mathbb{N}}$ is the function $M$, and therefore coincides, when using the finite volume scheme (11), with the H-limit of $\left(\mu_{n}\right)_{n \in \mathbb{N}}$; the use of some convergence for the weak $\star$ topology of $L^{\infty}(\Omega)$ is again sufficient to pass to the limit.

Remark 3 Note that the coincidence of the discrete and the continuous H-limits is not true for all the one-dimensional numerical schemes which can be associated with the same function $\mu$. Indeed, assume, in order to simplify, that $y_{k}-y_{k-1}=h$, for $k=1, \ldots, p$ (with $\left.h=1 / p\right), x_{k}=\left(y_{k}+y_{k-1}\right) / 2$, for $k=1, \ldots, p$, and that the function $\mu$ takes constant values in $\left(0, x_{1}\right), \ldots,\left(x_{k}, x_{k+1}\right), \ldots,\left(x_{p}, 1\right)$ which are $\mu_{r}$ and $\mu_{b}$ in alternance. If we discretize the Dirichlet problem with this function $\mu$ as diffusion coefficient and the piecewise linear finite element scheme with nodes located at the points $\left(y_{k}\right)_{k=0, \ldots, p}$, we obtain an approximate solution which is exactly the same as the one which is obtained by the same method (piecewise linear finite element) and a constant value of $\mu$ as diffusion coefficient, namely the arithmetic average of $\mu_{r}$ and $\mu_{b}$. Then, this approximate solution converges, as $h \longrightarrow 0$, towards the solution of the Dirichlet problem whose diffusion is this arithmetic average of $\mu_{r}$ and $\mu_{b}$. However, the H-limit as $h \longrightarrow 0$ of the continuous operators is given by the harmonic average of $\mu_{r}$ and $\mu_{b}$.

However, in the case $N>1$ and even in the isotropic case, the obtention of the Hd-limit by passing to the limit for the weak $\star$ topology of $L^{\infty}(\Omega)$ is no longer possible. Indeed, let us consider the sequence of admissible discretizations $\mathcal{D}_{n}$ of $\Omega=(0,1) \times(0,1)$, where the control volumes are some $(k / n,(k+$ $1) / n) \times(l / n,(l+1) / n)$, for integer values $k$ and $l$ between 0 and $n-1$ (see Figure 3). Assume that the function $\mu_{n}$ is defined by the value $\mu_{r}>0$ on the vertical edges $\{k / n\} \times(l / n,(l+1) / n)$ and by the value $\mu_{b}>0$ on the horizontal edges $(k / n,(k+1) / n) \times\{l / n\}$. Then the function $\mu_{n} \in \mathcal{M}_{\mathcal{D}_{n}}(\alpha, \beta)$ (with $\alpha=\min \left(\mu_{r}, \mu_{b}\right)$ and $\beta=\max \left(\mu_{r}, \mu_{b}\right)$ ) corresponds to the first two dimensional example of Section 2.4 (recall that the function $\mu_{n}$ is constant on subsets which, in this case, are the squares of lengthside equal to $1 /(n \sqrt{2})$, tilted with an angle of measure $\pi / 4$ with respect to the grid. As seen in Section 2 , the H-limit of $\left(\mu_{n}\right)_{n \in \mathbb{N}}$ is the field with constant value $\sqrt{\mu_{r} \mu_{b}}$. We then remark that for a given $f \in L^{2}(\Omega)$, the discrete values solution of the finite volume scheme (11) are identical to those obtained from (11), written in the case where $\tilde{\Omega}=\left(0,1 / \sqrt{\mu_{r}}\right) \times\left(0,1 / \sqrt{\mu_{b}}\right)$, the grid is given by the subsets $\left(k /\left(n \sqrt{\mu_{r}}\right),(k+\right.$ 1) $\left./\left(n \sqrt{\mu_{r}}\right)\right) \times\left(l /\left(n \sqrt{\mu_{b}}\right),(l+1) /\left(n \sqrt{\mu_{b}}\right)\right), \mu=1$, and the right hand side $\tilde{f}=f\left(\cdot \sqrt{\mu_{r}}, \cdot \sqrt{\mu_{b}}\right)$. Thanks to Lemma 3 which states the convergence of the finite volume scheme for $\mu=1$ we then get that $u_{\mathcal{D}_{n}}$ converges to $\bar{u}=\tilde{u}\left(\cdot / \sqrt{\mu_{r}}, \cdot / \sqrt{\mu_{b}}\right)$ with $\tilde{u}=\mathcal{F}_{\tilde{\Omega}}(\tilde{f}, 1)$, denoting here by $\mathcal{F}_{\tilde{\Omega}}$ the function $\mathcal{F}$ obtained when the Dirichlet problem is solved in the domain $\tilde{\Omega}$. An easy change of variable proves that $\bar{u}=\mathcal{F}(f, M)$, where $M$ is the constant field, the value of which is the linear application defined by $(1,0) \rightarrow\left(\mu_{r}, 0\right)$ and $(0,1) \rightarrow\left(0, \mu_{b}\right)$. This field $M$, which is homogeneous anisotropic and differs from the H-limit of $\left(\mu_{n}\right)_{n \in \mathbb{N}}$, is therefore the Hd-limit of $\left(\mathcal{D}_{n}, \mu_{n}\right)_{n \in \mathbb{N}}$.

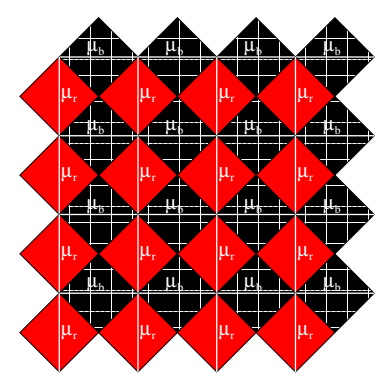

Figure 3: Case of discrepancy between the H- and Hd-limits

The physical reason of this discrepancy is the fact that in this example, the heterogeneous behavior and the grid are at the same scale: note that this occurs when solving the coupled two-phase flow in porous media problem using a coupled scheme on the same grid (see Section 5). On the contrary, in the cases where it is possible to let the size of the mesh tend to zero faster than the size of the heterogenities, the obtained H- and Hd-limits are equal. 
Remark 4 Similar results to Theorem 2 can be obtained within the finite element framework, leading to the same distinction between the resulting Hd-limit and the H-limit (see Remark 3 for an example in the one-dimensional case).

Remark 5 Exactly in the same manner as for the continuous case, it is possible to show the local character of Hd-convergence in the sense of Theorem 2, and the independence of the Hd-limit on the boundary conditions (see [16] for such results within the finite difference setting).

\subsection{Existence of limit operators}

The first step in direction to the proof of Theorem 2 is given by the results of the following lemma, which are similar to the continuous ones (see [14], [19]).

Lemma 4 Let $\Omega$ be an open bounded polygonal subset of $\mathbb{R}^{N}$, with $N \in \mathbb{N}_{*}$. Let two real numbers $\alpha$ and $\beta$ be such that $0<\alpha \leq \beta$. Let $\left(\mathcal{D}_{n}, \mu_{n}\right)_{n \in \mathbb{N}}$ be a sequence such that, for all $n \in \mathbb{N}$, $\mathcal{D}_{n}$ is an admissible discretization of $\Omega$ in the sense of Definition 1, and $\mu_{n} \in \mathcal{M}_{\mathcal{D}_{n}}(\alpha, \beta)$. We assume that $\lim _{n \longrightarrow \infty} \operatorname{size}\left(\mathcal{D}_{n}\right)=0$.

Then there exists a subsequence of $\left(\mathcal{D}_{n}, \mu_{n}\right)_{n \in \mathbb{N}}$, again denoted $\left(\mathcal{D}_{n}, \mu_{n}\right)_{n \in \mathbb{N}}$, that verifies: there exists an invertible continuous linear application $\bar{F}: H^{-1}(\Omega) \longrightarrow H_{0}^{1}(\Omega)$ and a continuous linear application $\overline{\mathbf{G}}: H^{-1}(\Omega) \longrightarrow\left(L^{2}(\Omega)\right)^{N}$ such that

- For all $f \in L^{2}(\Omega)$, the sequence $\left(F_{\mathcal{D}_{n}}\left(f, \mu_{n}\right)\right)_{n \in \mathbb{N}}$ converges to $\bar{F}(f)$ in $L^{2}(\Omega)$ and the sequence $\left(\mathbf{G}_{\mathcal{D}_{n}} F_{\mathcal{D}_{n}}\left(f, \mu_{n}\right)\right)_{n \in \mathbb{N}}$ weakly converges to $\nabla \bar{F}(f)$ in $\left(L^{2}(\Omega)\right)^{N}$,

- For all $f \in L^{2}(\Omega)$, the sequence $\left(\mu_{n} \mathbf{G}_{\mathcal{D}_{n}} F_{\mathcal{D}_{n}}\left(f, \mu_{n}\right)\right)_{n \in \mathbb{N}}$ weakly converges to $\overline{\mathbf{G}}(f)$ in $\left(L^{2}(\Omega)\right)^{N}$,

- The following relation holds

$$
\int_{\Omega} \overline{\mathbf{G}}(b)(x) \cdot \nabla \bar{v}(x) d x=b(\bar{v}), \forall \bar{v} \in H_{0}^{1}(\Omega), \forall b \in H^{-1}(\Omega) .
$$

Proof Let us assume the hypotheses of the lemma. Let $f \in L^{2}(\Omega)$. Thanks to (13), for all $n \in \mathbb{N}$, denoting $u_{n}=F_{\mathcal{D}_{n}}\left(f, \mu_{n}\right)$, we have

$$
\alpha\left|u_{n}\right|_{\mathcal{D}_{n}} \leq \operatorname{diam}(\Omega)\|f\|_{L^{2}(\Omega)} .
$$

This shows that the hypotheses of Lemma 2 are satisfied. Therefore, there exists a subsequence of $\left(\mathcal{D}_{n}, \mu_{n}\right)_{n \in \mathbb{N}}$, again denoted $\left(\mathcal{D}_{n}, \mu_{n}\right)_{n \in \mathbb{N}}$, and $\bar{u} \in H_{0}^{1}(\Omega)$ such that the sequence $\left(F_{\mathcal{D}_{n}}\left(f, \mu_{n}\right)\right)_{n \in \mathbb{N}}$ converges to $\bar{u}$ in $L^{2}(\Omega)$. We again denote $u_{n}=F_{\mathcal{D}_{n}}\left(f, \mu_{n}\right)$.

Let us introduce the functions $\bar{w} \in H_{0}^{1}(\Omega)$ defined by $\bar{w}=\mathcal{F}(f, 1)$ and, for all $n \in \mathbb{N}, w_{n}=F_{\mathcal{D}_{n}}(f, 1)$. For $n \in \mathbb{N}$, we deduce from (12) that

$$
\int_{\Omega} f(x) u_{n}(x) d x=\left[u_{n}, u_{n}\right]_{\mathcal{D}_{n}, \mu_{n}, 1} \geq \alpha\left(\left|u_{n}\right|_{\mathcal{D}_{n}}\right)^{2}
$$

and, thanks to the Cauchy-Schwarz inequality,

$$
\int_{\Omega} f(x) u_{n}(x) d x=\left[w_{n}, u_{n}\right]_{\mathcal{D}_{n}, 1,1} \leq\left|w_{n}\right|_{\mathcal{D}_{n}}\left|u_{n}\right|_{\mathcal{D}_{n}} .
$$

Therefore (18) and (19) yield

$$
\alpha\left|u_{n}\right|_{\mathcal{D}_{n}} \leq\left|w_{n}\right|_{\mathcal{D}_{n}} .
$$

Passing to the limit on $n \longrightarrow \infty$ in the above equation gives, using (14), 


$$
\alpha \limsup _{n \longrightarrow \infty}\left|u_{n}\right|_{\mathcal{D}_{n}} \leq\|\nabla \bar{w}\|_{\left(L^{2}(\Omega)\right)^{N}}
$$

which gives, since $\|f\|_{H^{-1}(\Omega)}=\|\nabla \bar{w}\|_{\left(L^{2}(\Omega)\right)^{N}}$,

$$
\alpha \limsup _{n \longrightarrow \infty}\left|u_{n}\right|_{\mathcal{D}_{n}} \leq\|f\|_{H^{-1}(\Omega)} .
$$

Thanks to (9), we get

$$
\alpha\|\bar{u}\|_{H_{0}^{1}(\Omega)} \leq\|f\|_{H^{-1}(\Omega)} .
$$

Turning to the study of the sequence $\mathbf{g}_{n}=\mu_{n} \mathbf{G}_{\mathcal{D}_{n}} F_{\mathcal{D}_{n}}\left(f, \mu_{n}\right)$ for $n \in \mathbb{N}$, we have (in a similar way as the case $\mu_{n}=1$ handled in Lemma 2),

$$
\left(\left\|\mathbf{g}_{n}\right\|_{\left(L^{2}(\Omega)\right)^{N}}\right)^{2} \leq N \beta^{2}\left(\left|u_{n}\right|_{\mathcal{D}_{n}}\right)^{2},
$$

which yields, using (20),

$$
\limsup _{n \longrightarrow \infty}\left\|\mathbf{g}_{n}\right\|_{\left(L^{2}(\Omega)\right)^{N}} \leq \frac{\sqrt{N} \beta}{\alpha}\|f\|_{H^{-1}(\Omega)} .
$$

Thus there exists a subsequence of $\left(\mathcal{D}_{n}, \mu_{n}\right)_{n \in \mathbb{N}}$, again denoted $\left(\mathcal{D}_{n}, \mu_{n}\right)_{n \in \mathbb{N}}$ and $\overline{\mathbf{g}} \in\left(L^{2}(\Omega)\right)^{N}$ such that $\mathbf{g}_{n}=\mu_{n} \mathbf{G}_{\mathcal{D}_{n}} F_{\mathcal{D}_{n}}\left(f, \mu_{n}\right)$ converges weakly to $\overline{\mathbf{g}}$ as $n \longrightarrow \infty$ in $\left(L^{2}(\Omega)\right)^{N}$. Passing to the limit in (22), we then get

$$
\|\overline{\mathbf{g}}\|_{\left(L^{2}(\Omega)\right)^{N}} \leq \frac{\sqrt{N} \beta}{\alpha}\|f\|_{H^{-1}(\Omega)} .
$$

We then consider a sequence $\left(f_{m}\right)_{m \in \mathbb{N}}$ of functions of $L^{2}(\Omega)$ which is dense in $H^{-1}(\Omega)$. We can then extract a subsequence (using the classical diagonal process) again denoted $\left(\mathcal{D}_{n}, \mu_{n}\right)_{n \in \mathbb{N}}$, such that for all $m \in \mathbb{N}$ the sequence $\left(F\left(f_{m}, \mathcal{D}_{n}, \mu_{n}\right)\right)_{n \in \mathbb{N}}$ converges to some function denoted $\bar{F}\left(f_{m}\right) \in H_{0}^{1}(\Omega)$ in $L^{2}(\Omega)$ and the sequence $\left(\mu_{n} \mathbf{G}_{\mathcal{D}_{n}} F\left(f_{m}, \mathcal{D}_{n}, \mu_{n}\right)\right)_{n \in \mathbb{N}}$ converges to some function denoted $\overline{\mathbf{G}}\left(f_{m}\right) \in\left(L^{2}(\Omega)^{N}\right)$ weakly in $\left(L^{2}(\Omega)^{N}\right)$. The linear functions $\bar{F}$ (resp. $\overline{\mathbf{G}}$ ) can then be prolonged by continuity, thanks to $(21)$ (resp. $(23)$ ) to a continuous linear function, again denoted $\bar{F}: H^{-1}(\Omega) \longrightarrow H_{0}^{1}(\Omega)$ (resp. $\left.\overline{\mathbf{G}}: H^{-1}(\Omega) \longrightarrow\left(L^{2}(\Omega)\right)^{N}\right)$.

Let us now prove (16). Let $f \in L^{2}(\Omega)$. We set $\bar{u}=\bar{F}(f)$ and $\overline{\mathbf{g}}=\overline{\mathbf{G}}(f)$. Let $\varphi \in C_{c}^{\infty}(\Omega)$. For a given $n \in \mathbb{N}$, we denote $\mathcal{D}_{n}=\left(\mathcal{T}_{n}, \mathcal{E}_{n}, \mathcal{P}_{n}\right), u_{n}=F_{\mathcal{D}_{n}}\left(f, \mu_{n}\right)$. Omitting the indexes $n$ in the discrete expressions, we set, for all $K \in \mathcal{T}$ and $\sigma \in \mathcal{E}_{K}$,

$$
R_{K, \sigma}=\frac{1}{d_{K, \sigma}}\left(\varphi\left(y_{\sigma}\right)-\varphi\left(x_{K}\right)\right)-\frac{N}{m_{\sigma} d_{K, \sigma}} \int_{D_{K, \sigma}} \nabla \varphi(x) \cdot \mathbf{n}_{K, \sigma} d x .
$$

Then there exists $C_{\varphi}>0$ which only depends on $\varphi$ such that $\left|R_{K, \sigma}\right| \leq \operatorname{size}\left(\mathcal{D}_{n}\right) C_{\varphi}$. Setting

$$
T_{n}=\sum_{K \in \mathcal{T}_{n}} \sum_{\sigma \in \mathcal{E}_{K}} \mu_{\sigma} \tau_{K, \sigma}\left(u_{\sigma}-u_{K}\right)\left(\varphi\left(y_{\sigma}\right)-\varphi\left(x_{K}\right)\right),
$$

we then get $\lim _{n \longrightarrow \infty}\left|T_{n}-\int_{\Omega} \mathbf{g}_{n}(x) \cdot \nabla \varphi(x) d x\right|=0$ which yields

$$
\lim _{n \longrightarrow \infty} T_{n}=\int_{\Omega} \overline{\mathbf{g}}(x) \cdot \nabla \varphi(x) d x .
$$

Since, using (11), we have

$$
T_{n}=\sum_{K \in \mathcal{T}_{n}} \int_{K} f(x) d x \varphi\left(x_{K}\right)
$$


we also get $\lim _{n \longrightarrow \infty} T_{n}=\int_{\Omega} f(x) \varphi(x) d x$. We thus get

$$
\int_{\Omega} \overline{\mathbf{g}}(x) \cdot \nabla \varphi(x) d x=\int_{\Omega} f(x) \varphi(x) d x, \forall \varphi \in C_{c}^{\infty}(\Omega) .
$$

Using (26) and the density of $C_{c}^{\infty}(\Omega)$ in $H_{0}^{1}(\Omega)$, we conclude (16).

Let us show that $\bar{F}$ is invertible. We consider the bilinear form $a:\left(H^{-1}(\Omega)\right)^{2} \longrightarrow \mathbb{R}$ defined by

$$
\forall\left(b, b^{\prime}\right) \in\left(H^{-1}(\Omega)\right)^{2}, a\left(b, b^{\prime}\right)=b\left(\bar{F}\left(b^{\prime}\right)\right) .
$$

Let again $f \in L^{2}(\Omega)$. We introduce the functions $\bar{u}, \bar{w} \in H_{0}^{1}(\Omega)$ defined by $\bar{u}=\bar{F}(f), \bar{w}=\mathcal{F}(f, 1)$ and, for all $n \in \mathbb{N}, u_{n}=F_{\mathcal{D}_{n}}\left(f, \mu_{n}\right)$ and $w_{n}=F_{\mathcal{D}_{n}}(f, 1)$. We have

$$
a(f, f)=\int_{\Omega} f(x) \bar{F}(f)(x) d x=\lim _{n \longrightarrow \infty} \int_{\Omega} f(x) u_{n}(x) d x .
$$

We can write, on the one hand,

$$
\int_{\Omega} f(x) u_{n}(x) d x=\left[u_{n}, u_{n}\right]_{\mathcal{D}_{n}, \mu_{n}, 1},
$$

which yields

$$
\int_{\Omega} f(x) u_{n}(x) d x \geq \alpha\left(\left|u_{n}\right|_{\mathcal{D}_{n}}\right)^{2} .
$$

We have, on the other hand,

$$
\int_{\Omega} f(x) w_{n}(x) d x=\left[u_{n}, w_{n}\right]_{\mathcal{D}_{n}, \mu_{n}, 1}
$$

and

$$
\int_{\Omega} f(x) w_{n}(x) d x=\left(\left|w_{n}\right|_{\mathcal{D}_{n}}\right)^{2} .
$$

This yields

$$
\left(\left|w_{n}\right|_{\mathcal{D}_{n}}\right)^{2}=\left[u_{n}, w_{n}\right]_{\mathcal{D}_{n}, \mu_{n}, 1}
$$

Since

$$
\begin{aligned}
\left(\left[u_{n}, w_{n}\right]_{\mathcal{D}_{n}, \mu_{n}, 1}\right)^{2} & \leq\left[u_{n}, u_{n}\right]_{\mathcal{D}_{n}, \mu_{n}, 1}\left[w_{n}, w_{n}\right]_{\mathcal{D}_{n}, \mu_{n}, 1} \\
& \leq \beta^{2}\left[u_{n}, u_{n}\right]_{\mathcal{D}_{n}, 1,1}\left[w_{n}, w_{n}\right]_{\mathcal{D}_{n}, 1,1}
\end{aligned}
$$

we therefore get

$$
\left(\left|w_{n}\right|_{\mathcal{D}_{n}}\right)^{2} \leq \beta\left|u_{n}\right|_{\mathcal{D}_{n}}\left|w_{n}\right|_{\mathcal{D}_{n}}
$$

From (27) and (28) we deduce

$$
\int_{\Omega} f(x) u_{n}(x) d x \geq \frac{\alpha}{\beta^{2}}\left(\left|w_{n}\right|_{\mathcal{D}_{n}}\right)^{2} .
$$

Letting $n \longrightarrow \infty$ in (29) gives

$$
a(f, f) \geq \frac{\alpha}{\beta^{2}} \int_{\Omega}(\nabla \bar{w}(x))^{2} d x
$$

which shows that 


$$
a(f, f) \geq \frac{\alpha}{\beta^{2}}\left(\|f\|_{H^{-1}(\Omega)}\right)^{2} .
$$

By continuity of $a$, this property is available on $H^{-1}(\Omega)$, which shows the coercivity of $a$. Let $\bar{v} \in H_{0}^{1}(\Omega)$. The problem: find $b \in H^{-1}(\Omega)$ such that for all $b^{\prime} \in H^{-1}(\Omega), a\left(b^{\prime}, b\right)=b^{\prime}(\bar{v})$, has a unique solution $b$, thanks to Lax-Milgram's theorem. It then satisfies $\bar{F}(b)=\bar{v}$.

\section{Remark 6}

The previous lemma could be stated using sequences $\left(F_{\mathcal{D}_{n}}\left(f, \mu_{n}\right)\right)_{n \in \mathbb{N}}$ and $\left(\mathbf{G}_{\mathcal{D}_{n}} F_{\mathcal{D}_{n}}\left(f, \mu_{n}\right)\right)_{n \in \mathbb{N}}$ with $f \in H^{-1}(\Omega)$ (see [6] for the definition of the finite volume scheme in this case).

\subsection{Proof of Theorem 2}

We assume the hypotheses of Theorem 2, which are the same as those of Lemma 4 . Therefore, let $\left(\mathcal{D}_{n}, \mu_{n}\right)_{n \in \mathbb{N}}$ denote a subsequence of $\left(\mathcal{D}_{n}, \mu_{n}\right)_{n \in \mathbb{N}}$, and $\bar{F}: H^{-1}(\Omega) \longrightarrow H_{0}^{1}(\Omega)$ and $\overline{\mathbf{G}}: H^{-1}(\Omega) \longrightarrow$ $\left(L^{2}(\Omega)^{N}\right)$ denote the linear continuous functions verifying the conclusions of Lemma 4 . It suffices now to prove that there exists a function $M: \Omega \longrightarrow \mathcal{L}\left(\mathbb{R}^{N}, \mathbb{R}^{N}\right)$ such that for a.e. $x \in \Omega, \overline{\mathbf{G}}(b)(x)=$ $M(x) \nabla \bar{F}(b)(x)$, for all $b \in H^{-1}(\Omega)$, and for all $(\xi, \chi) \in\left(\mathbb{R}^{N}\right)^{2}, \alpha|\xi|^{2} \leq M(x) \xi \cdot \xi \leq \beta|\xi|^{2}$, and $M(x) \xi \cdot \chi=$ $\xi \cdot M(x) \chi$. Let $f, g \in L^{2}(\Omega)$. We set $\bar{u}=\bar{F}(f)$ and $\bar{v}=\bar{F}(g)$. Let $\varphi \in C_{c}^{\infty}(\Omega)$. For a given $n \in \mathbb{N}$, we denote $\mathcal{D}_{n}=\left(\mathcal{T}_{n}, \mathcal{E}_{n}, \mathcal{P}_{n}\right), u_{n}=F_{\mathcal{D}_{n}}\left(f, \mu_{n}\right), v_{n}=F_{\mathcal{D}_{n}}\left(g, \mu_{n}\right)$, and we consider the expression

$$
A_{n}=\left[u_{n}, v_{n}\right]_{\mathcal{D}_{n}, \mu_{n}, \varphi} .
$$

We get $A_{n}=B_{n}-C_{n}$, where $B_{n}$ and $C_{n}$ are defined by (omitting indexes $n$ in the right hand sides)

$$
B_{n}=\sum_{K \in \mathcal{T}} \sum_{\sigma \in \mathcal{E}_{K}} \mu_{\sigma} \tau_{K, \sigma}\left(u_{\sigma}-u_{K}\right)\left(\varphi\left(y_{\sigma}\right) v_{\sigma}-\varphi\left(x_{K}\right) v_{K}\right)
$$

and

$$
C_{n}=\sum_{K \in \mathcal{T}} \sum_{\sigma \in \mathcal{E}_{K}} v_{\sigma} \mu_{\sigma} \tau_{K, \sigma}\left(u_{\sigma}-u_{K}\right)\left(\varphi\left(y_{\sigma}\right)-\varphi\left(x_{K}\right)\right) .
$$

Since $B_{n}=\sum_{K \in \mathcal{T}} \int_{K} f(x) d x \varphi\left(x_{K}\right) v_{K}$, we then get

$$
\lim _{n \longrightarrow \infty} B_{n}=\int_{\Omega} f(x) \varphi(x) \bar{v}(x) d x .
$$

Let $\tilde{v} \in C_{c}^{\infty}(\Omega)$ be a function which is meant to tend to $\bar{v}$ in $H_{0}^{1}(\Omega)$. We set

$$
\tilde{B}_{n}=\sum_{K \in \mathcal{T}} \sum_{\sigma \in \mathcal{E}_{K}} \mu_{\sigma} \tau_{K, \sigma}\left(u_{\sigma}-u_{K}\right)\left(\varphi\left(y_{\sigma}\right) \tilde{v}\left(y_{\sigma}\right)-\varphi\left(x_{K}\right) \tilde{v}\left(x_{K}\right)\right)
$$

and we again have $\tilde{B}_{n}=\sum_{K \in \mathcal{T}} \int_{K} f(x) d x \varphi\left(x_{K}\right) \tilde{v}\left(x_{K}\right)$, which yields

$$
\lim _{n \longrightarrow \infty} \tilde{B}_{n}=\int_{\Omega} f(x) \varphi(x) \tilde{v}(x) d x .
$$

Using (24), we have

$$
\lim _{n \longrightarrow \infty} \tilde{B}_{n}=\int_{\Omega} \overline{\mathbf{G}}(f)(x) \cdot \nabla(\varphi(x) \tilde{v}(x)) d x .
$$

We thus get

$$
\int_{\Omega} f(x) \varphi(x) \tilde{v}(x) d x=\int_{\Omega} \overline{\mathbf{G}}(f)(x) \cdot \nabla(\varphi(x) \tilde{v}(x)) d x .
$$


In (33), we let $\tilde{v} \longrightarrow \bar{v}$ in $H_{0}^{1}(\Omega)$. It gives

$$
\int_{\Omega} f(x) \varphi(x) \bar{v}(x) d x=\int_{\Omega} \overline{\mathbf{G}}(f)(x) \cdot \nabla(\varphi(x) \bar{v}(x)) d x
$$

and therefore

$$
\lim _{n \longrightarrow \infty} B_{n}=\int_{\Omega} \overline{\mathbf{G}}(f)(x) \cdot \nabla(\varphi(x) \bar{v}(x)) d x .
$$

We now study $C_{n}$. Let $\hat{v}_{n}$ be the function defined by

$$
\hat{v}_{n}(x)=v_{\sigma}, \forall x \in D_{K, \sigma}, \forall K \in \mathcal{T}, \forall \sigma \in \mathcal{E}_{K} .
$$

Since $\left|v_{n}\right|_{\mathcal{D}_{n}}$ remains bounded, it is easy to see that $\hat{v}_{n}-v_{n}$ converges to 0 in $L^{2}(\Omega)$. We set

$$
\hat{C}_{n}=\int_{\Omega} \hat{v}_{n}(x) \mu_{n} \mathbf{G}_{\mathcal{D}_{n}} F_{\mathcal{D}_{n}}\left(f, \mu_{n}\right)(x) \cdot \nabla \varphi(x) d x .
$$

We easily get, thanks to the Cauchy-Schwarz inequality,

$$
\left|C_{n}-\hat{C}_{n}\right| \leq C(\varphi, \beta) \operatorname{size}\left(\mathcal{D}_{n}\right)\left|u_{n}\right|_{\mathcal{D}_{n}}\left\|\hat{v}_{n}\right\|_{L^{2}(\Omega)},
$$

which shows that

$$
\lim _{n \longrightarrow \infty} C_{n}=\lim _{n \longrightarrow \infty} \hat{C}_{n}=\int_{\Omega} \bar{v}(x) \overline{\mathbf{G}}(f)(x) \cdot \nabla \varphi(x) d x .
$$

We thus get, gathering (35) and (37), recalling that $\bar{v}=\bar{F}(g)$,

$$
\lim _{n \longrightarrow \infty} A_{n}=\int_{\Omega} \varphi(x) \overline{\mathbf{G}}(f)(x) \cdot \nabla \bar{F}(g)(x) d x .
$$

Note that the preceding proof $((32)-(38))$ also gives a discrete version of a compensated compactness lemma, see Remark 7 below. We can now exchange the roles of $f$ and $g$ in (38). We thus get

$$
\lim _{n \longrightarrow \infty} A_{n}=\int_{\Omega} \varphi(x) \overline{\mathbf{G}}(g)(x) \cdot \nabla \bar{F}(f)(x) d x .
$$

This yields

$$
\int_{\Omega} \varphi(x) \overline{\mathbf{G}}(f)(x) \cdot \nabla \bar{F}(g)(x) d x=\int_{\Omega} \varphi(x) \overline{\mathbf{G}}(g)(x) \cdot \nabla \bar{F}(f)(x) d x .
$$

In order to prove the existence of $M$ as it is given in Theorem 2, we now proceed exactly as in the continuous setting. Since (40) is true for all $\varphi \in C_{c}^{\infty}(\Omega)$, we get

$$
\overline{\mathbf{G}}(f)(x) \cdot \nabla \bar{F}(g)(x)=\overline{\mathbf{G}}(g)(x) \cdot \nabla \bar{F}(f)(x) \text {, for a.e. } x \in \Omega .
$$

Since (41) is true for all $f$ and $g$ in $L^{2}(\Omega)$, by continuity of $\bar{F}$ and $\overline{\mathbf{G}}$, we get:

$$
\overline{\mathbf{G}}(b)(x) \cdot \nabla \bar{F}\left(b^{\prime}\right)(x)=\overline{\mathbf{G}}\left(b^{\prime}\right)(x) \cdot \nabla \bar{F}(b)(x), \forall b, b^{\prime} \in H^{-1}(\Omega), \text { for a.e. } x \in \Omega .
$$

Since $\bar{F}$ is invertible, we can choose some $b_{i} \in H^{-1}(\Omega)$ such that, in an open set $\omega$ such that $\bar{\omega} \subset \Omega$, $\bar{F}\left(b_{i}\right)(x)=x \cdot \mathbf{e}_{i}$ and then $\nabla \bar{F}\left(b_{i}\right)(x)=\mathbf{e}_{i}$ (where $\mathbf{e}_{i}$ is the $i$ th unit vector of $\mathbb{R}^{N}$ ), for $i=1, \ldots, N$. Thus, for a.e. $x \in \omega$, we can define $M(x) \in \mathcal{L}\left(\mathbb{R}^{N}, \mathbb{R}^{N}\right)$ by $M^{\star}(x) \mathbf{e}_{i}=\overline{\mathbf{G}}\left(b_{i}\right)(x)$, for $i=1, \ldots, N$, where $M^{\star}$ is the adjoint operator of $M$. We thus get

$$
\overline{\mathbf{G}}(b)(x)=M(x) \nabla \bar{F}(b)(x), \forall b \in H^{-1}(\Omega) \text { for a.e. } x \in \omega .
$$


Taking $b=b_{i}$ and $b^{\prime}=b_{j}$ in (42) proves that $M(x)$ is symmetric in $\omega$. Since $\omega$ is arbitrary, we then obtain $M$ a.e. in $\Omega$ such that

$$
\overline{\mathbf{G}}(b)(x)=M(x) \nabla \bar{F}(b)(x), \forall b \in H^{-1}(\Omega) \text { for a.e. } x \in \Omega .
$$

The uniqueness of $M$ is a direct consequence of the invertibility of $\bar{F}$. We now prove that $M \in \mathcal{M}(\alpha, \beta, \Omega)$. Letting $f=g$ in (32), and taking $\varphi \geq 0$, we get, using (38), that

$$
\lim _{n \longrightarrow \infty}\left[u_{n}, u_{n}\right]_{\mathcal{D}_{n}, \mu_{n}, \varphi}=\int_{\Omega} \varphi(x) M(x) \nabla \bar{u}(x) \cdot \nabla \bar{u}(x) d x .
$$

Since

$$
\begin{aligned}
\lim _{n \longrightarrow \infty}\left[u_{n}, u_{n}\right]_{\mathcal{D}_{n}, \mu_{n}, \varphi} & \geq \alpha \liminf _{n \longrightarrow \infty}\left[u_{n}, u_{n}\right]_{\mathcal{D}_{n}, 1, \varphi} \\
& \geq \alpha \int_{\Omega} \varphi(x)(\nabla \bar{u}(x))^{2} d x,
\end{aligned}
$$

we get, for a.e. $x \in \Omega, M(x) \nabla \bar{F}(f)(x) \cdot \nabla \bar{F}(f)(x) \geq \alpha(\nabla \bar{F}(f)(x))^{2}$. By density and invertibility of $\bar{F}$, since $f$ can be arbitrarily chosen, this proves that, for a.e. $x \in \Omega$ and for all $\xi \in \mathbb{R}^{N}, M(x) \xi \cdot \xi \geq \alpha(\xi)^{2}$. Let $\varphi \in C_{c}^{\infty}\left(\Omega, \mathbb{R}_{+}\right)$, and $(f, g) \in\left(L^{2}(\Omega)\right)^{N}, \bar{u}=\bar{F}(f)$ and $\bar{w}=\mathcal{F}(f, 1)$. For $n \in \mathbb{N}$, we define $u_{n}=F_{\mathcal{D}_{n}}\left(f, \mu_{n}\right)$ and $w_{n}=F_{\mathcal{D}_{n}}(g, 1)$. We define $D_{n}$ by

$$
D_{n}=\left[u_{n}, w_{n}\right]_{\mathcal{D}_{n}, \mu_{n}, \varphi}
$$

We study $D_{n}$ in the same manner as $A_{n}$. Since $w_{n}$ converges to $\bar{w}$ in $L^{2}(\Omega)$, we get

$$
\lim _{n \longrightarrow \infty} D_{n}=\int_{\Omega} \varphi(x) M(x) \nabla \bar{u}(x) \cdot \nabla \bar{w}(x) d x .
$$

On the other hand, we have

$$
\left(D_{n}\right)^{2} \leq\left[u_{n}, u_{n}\right]_{\mathcal{D}_{n}, \mu_{n}, \varphi}\left[w_{n}, w_{n}\right]_{\mathcal{D}_{n}, \mu_{n}, \varphi}
$$

and therefore

$$
\left(D_{n}\right)^{2} \leq \beta\left[u_{n}, u_{n}\right]_{\mathcal{D}_{n}, \mu_{n}, \varphi}\left[w_{n}, w_{n}\right]_{\mathcal{D}_{n}, 1, \varphi}
$$

We thus get

$$
\lim _{n \longrightarrow \infty}\left(D_{n}\right)^{2} \leq \beta \int_{\Omega} \varphi(x) M(x) \nabla \bar{u}(x) \cdot \nabla \bar{u}(x) d x \quad \int_{\Omega} \varphi(x)(\nabla \bar{w}(x))^{2} d x
$$

which gives

$$
\left(\int_{\Omega} \varphi(x) M(x) \nabla \bar{u}(x) \cdot \nabla \bar{w}(x) d x\right)^{2} \leq \beta \quad \int_{\Omega} \varphi(x) M(x) \nabla \bar{u}(x) \cdot \nabla \bar{u}(x) d x \quad \int_{\Omega} \varphi(x)(\nabla \bar{w}(x))^{2} d x .
$$

Since $g$ can be arbitrarily chosen, it is therefore possible to let $\bar{w} \longrightarrow \bar{u}$ (in $H_{0}^{1}(\Omega)$ ) in (48). We thus get

$$
\int_{\Omega} \varphi(x) M(x) \nabla \bar{u}(x) \cdot \nabla \bar{u}(x) d x \leq \beta \quad \int_{\Omega} \varphi(x)(\nabla \bar{u}(x))^{2} d x,
$$

which yields, for a.e. $x \in \Omega, M(x) \nabla \bar{u}(x) \cdot \nabla \bar{u}(x) \leq \beta(\nabla \bar{u}(x))^{2}$. By density and invertibility of $\bar{F}$, since $f$ can be arbitrarily chosen, we get that, for a.e. $x \in \Omega$ and for all $\xi \in \mathbb{R}^{N}, M(x) \xi \cdot \xi \leq \beta \xi^{2}$.

This concludes the proof of Theorem 2 . 
Remark 7 Note that, as in the continuous setting (see [14] and [19]) and as in the finite difference framework (see [12] and [16]), an important step of the above proof (from (32) to (38)) consists in passing to the limit in some nonlinear terms. Indeed, the same proof as above also yields the following discrete version of a compensated compactness lemma (namely a discrete simplified "div-curl" lemma):

Lemma 5 (Discrete compensated compactness lemma) Let $\Omega$ be an open bounded polygonal subset of $\mathbb{R}^{N}$, with $N \in \mathbb{N}_{*}$. Let $\left(\mathcal{D}_{n}\right)_{n \in \mathbb{N}}$ be a sequence of admissible discretizations of $\Omega$ in the sense of Definition 1 such that $\lim _{n \rightarrow \infty} \operatorname{size}\left(\mathcal{D}_{n}\right)=0$. Let us suppose that for all $n \in \mathbb{N}$, there exists $W_{n}, X_{n} \in$ $V_{\mathcal{D}_{n}} \subset\left(L^{2}(\Omega)\right)^{N}$ such that

- $W_{n} \longrightarrow W$ weakly in $\left(L^{2}(\Omega)\right)^{N}$ as $n \longrightarrow \infty$,

- $X_{n} \longrightarrow X$ weakly in $\left(L^{2}(\Omega)\right)^{N}$ as $n \longrightarrow \infty$,

- $\operatorname{div}_{\mathcal{D}_{n}} W_{n}$ weakly converges in $L^{2}(\Omega)$ as $n \longrightarrow \infty$,

- there exists $u_{n} \in H_{\mathcal{D}_{n}}(\Omega)$ such that $X_{n}=\mathbf{G}_{\mathcal{D}_{n}} u_{n}$.

Then $\lim _{n \longrightarrow \infty}\left\langle W_{n}, G_{n}\right\rangle_{\mathcal{D}_{n}}=\int_{\Omega} W(x) \cdot X(x) d x$.

In the preceding lemma, we denote, for any admissible discretization $\mathcal{D}$ of $\Omega$ in the sense of Definition 1, by $V_{\mathcal{D}}$ the subset of $\left(L^{2}(\Omega)\right)^{N}$ of functions $W$ verifying that there exists a family of real values $\left(w_{K, \sigma}\right)_{K \in \mathcal{T}, \sigma \in \mathcal{E}_{K}}$ such that

$$
W(x)=N w_{K, \sigma} \mathbf{n}_{K, \sigma}, \text { for a.e. } x \in D_{K, \sigma}, \forall K \in \mathcal{T}, \forall \sigma \in \mathcal{E}_{K},
$$

and $w_{K, \sigma}+w_{L, \sigma}=0$ for all $\sigma=K \mid L$.

For all $W \in V_{\mathcal{D}}$, we denote by $\operatorname{div}_{\mathcal{D}} W$ the piecewise constant function, whose value in $K \in \mathcal{T}$ is $\sum_{\sigma \in \mathcal{E}_{K}} m_{\sigma} w_{K, \sigma}$. We then get $\mathbf{G}_{\mathcal{D}} u \in V_{\mathcal{D}}$ for all $u \in H_{\mathcal{D}}(\Omega)$. For $(W, X) \in\left(V_{\mathcal{D}}\right)^{2}$, we then define $\langle W, X\rangle_{\mathcal{D}}=\frac{1}{N} \int_{\Omega} W(x) \cdot X(x) d x$. It is interesting to notice that, under the hypotheses of the lemma, neither the sequence $\left(W_{n}\right)_{n \in \mathbb{N}}$ nor $\left(X_{n}\right)_{n \in \mathbb{N}}$ converge in $\left(L^{2}(\Omega)\right)^{N}$, except if the limit is 0 . Note also that, contrary to the classical compensated compactness lemma, the sequence which converges in the distribution sense to $W \cdot X$ is not $\left(W_{n} \cdot X_{n}\right)_{n \in \mathbb{N}}$ but it is $\frac{1}{N}\left(W_{n} \cdot X_{n}\right)_{n \in \mathbb{N}}$.

\section{Application to a coupled problem}

\subsection{A continuous system of equations}

We now study the steady-state version of the evolution problem (1). We thus get the following system

$$
\left.\begin{array}{ll}
-\operatorname{div}(\lambda(s) \nabla u) & =\bar{f} \\
-\operatorname{div}(\gamma(s) \lambda(s) \nabla u) & =(\bar{f})^{+} \gamma(\bar{s})-(\bar{f})^{-} \gamma(s)
\end{array}\right\} \text { in } \Omega,
$$

with the boundary conditions

$$
\begin{aligned}
& u=0 \text { on } \partial \Omega, \\
& s=\hat{s} \text { on }\left\{x \in \partial \Omega, \nabla u(x) \cdot \mathbf{n}_{\partial \Omega}(x) \geq 0\right\} .
\end{aligned}
$$

We refer to the introduction for the physical meaning of the quantities appearing in (50) and (51). The following assumptions (denoted in the following Hypotheses $(\mathrm{H})$ ) are made on the data:

- the domain $\Omega$ is an open polygonal connex subset of $\mathbb{R}^{N}$, with $N=2$ or $N=3$, 
- $\gamma \in C^{0}([0,1],[0,1])$ is a nondecreasing Lipschitz continuous function with $\gamma(0)=0$ and $\gamma(1)=1$, and Lipschitz constant $L_{\gamma}>0$,

- there exists two real numbers $\alpha$ and $\beta$, with $0<\alpha \leq \beta$ such that $\lambda \in C^{0}([0,1],[\alpha, \beta])$ (recall that $\lambda$ is the "total mobility") verifies that $\gamma \lambda$ (the mobility of the phase 1 , also denoted below $k_{1}$ ) is nondecreasing and $(1-\gamma) \lambda$ (the mobility of the phase 2 ) is nonincreasing,

- $\bar{f} \in L^{2}(\Omega)$ represents the rates at the wells,

- $\bar{s} \in L^{\infty}(\Omega)$ is such that $0 \leq \bar{s} \leq 1$ a.e. in $\Omega$,

- $\hat{s} \in L^{\infty}(\partial \Omega)$ is such that $0 \leq \hat{s} \leq 1$ a.e. in $\partial \Omega$ (for the $N-1$ dimensional Lebesgue measure).

\subsection{Finite volume coupled scheme}

Let us assume Hypotheses (H). Let $\mathcal{D}$ be an admissible discretization of $\Omega$ in the sense of Definition 1 . We set

$$
\left.\begin{array}{ll}
\bar{f}_{K}=\int_{K} \bar{f}(x) d x, \bar{s}_{K}=\frac{1}{m_{K}} \int_{K} \bar{s}(x) d x, & \forall K \in \mathcal{T}, \\
\hat{s}_{\sigma}=\frac{1}{m_{\sigma}} \int_{\sigma} \hat{s}(x) d x, & \forall \sigma \in \mathcal{E}_{\text {ext }} .
\end{array}\right\}
$$

We introduce the set $L_{\mathcal{D}}(\Omega,[0,1])$ of the functions of $L^{\infty}(\Omega)$ whose value on each $K \in \mathcal{T}$ is a constant value belonging to $[0,1]$. For all $s \in L_{\mathcal{D}}(\Omega,[0,1])$ and $K \in \mathcal{T}$, we denote $s_{K} \in[0,1]$ the constant value of $s$ in $K$. For all $u \in H_{\mathcal{D}}(\Omega)$ and $s \in L_{\mathcal{D}}(\Omega,[0,1])$, the upstream evaluation of the saturation at the edges $\sigma \in \mathcal{E}$ is defined by the functions $s_{\sigma}(u, s, \hat{s})$ such that

$$
\begin{aligned}
& \left.\begin{array}{ll}
s_{\sigma}(u, s, \hat{s})=s_{K} & \text { if } u_{K} \geq u_{L} \\
s_{\sigma}(u, s, \hat{s})=s_{L} & \text { if } u_{K}<u_{L}
\end{array}\right\} \quad \forall \sigma \in \mathcal{E}_{\text {int }}, \sigma=K \mid L \\
& \left.\begin{array}{ll}
s_{\sigma}(u, s, \hat{s})=s_{K} & \text { if } u_{K} \geq 0 \\
s_{\sigma}(u, s, \hat{s})=\hat{s}_{\sigma} & \text { if } u_{K}<0
\end{array}\right\} \quad \forall \sigma \in \mathcal{E}_{\text {ext }}, \sigma \in \mathcal{E}_{K},
\end{aligned}
$$

and the functions $\mu(u, s, \hat{s}) \in \mathcal{M}_{\mathcal{D}}(\alpha, \beta)$ by

$$
\mu_{\sigma}(u, s, \hat{s})=\lambda\left(s_{\sigma}(u, s, \hat{s})\right), \forall \sigma \in \mathcal{E} .
$$

We consider the following scheme (classical in petroleum engineering), a solution of which is some $(u, s) \in$ $H_{\mathcal{D}}(\Omega) \times L_{\mathcal{D}}(\Omega,[0,1]):$

$$
\begin{gathered}
u=F(\bar{f}, \mathcal{D}, \mu(u, s, \hat{s})), \\
-\sum_{\sigma \in \mathcal{E}_{K}} \gamma\left(s_{\sigma}(u, s, \hat{s})\right) \mu_{\sigma}(u, s, \hat{s}) \tau_{K, \sigma}\left(u_{\sigma}-u_{K}\right)=\gamma\left(\bar{s}_{K}\right)\left(\bar{f}_{K}\right)^{+}-\gamma\left(s_{K}\right)\left(\bar{f}_{K}\right)^{-}, \quad \forall K \in \mathcal{T} .
\end{gathered}
$$

Remark 8 Note that the function $\lambda$ is also evaluated in (54) using an upstream weighted scheme. This corresponds to the industrial scheme classicaly used in reservoir simulation, in which the mobility of each phase is upstream weighted. However, it would be natural to use a centered approximation in (54) and use an upstream weighted scheme for $\gamma$ in the left hand side of (56), but in such a case the convergence results given in Theorem 4 should be weakened. 


\subsubsection{Existence of a solution to the coupled scheme}

Lemma 6 Let us assume Hypotheses $(H)$. Let $\mathcal{D}$ be an admissible discretization of $\Omega$ in the sense of Definition 1. Then there exists at least one solution $(u, s) \in H_{\mathcal{D}}(\Omega) \times L_{\mathcal{D}}(\Omega,[0,1])$ to Scheme (52)-(56).

Proof We prove the existence of a solution of (52)-(56) using Brouwer's fixed point theorem. For all $K \in \mathcal{T}$, let us define $\bar{f}_{K}, \bar{s}_{K}, \hat{s}_{K}$ by $(52)$. We denote by

$$
E=\left\{u \in H_{\mathcal{D}}(\Omega), \alpha|u|_{\mathcal{D}} \leq \operatorname{diam}(\Omega)\|\bar{f}\|_{L^{2}(\Omega)}\right\} .
$$

We define the application $\mathcal{A}: E \times L_{\mathcal{D}}(\Omega,[0,1]) \longrightarrow E \times L_{\mathcal{D}}(\Omega,[0,1])$ by $\mathcal{A}(u, s)=\left(u^{\prime}, s^{\prime}\right)$, with:

- $u^{\prime}=F(\bar{f}, \mathcal{D}, \mu(u, s, \hat{s}))$,

- for a real value $k>0$ which will be chosen later, we define $\left(s_{K}^{\prime}\right)_{K \in \mathcal{T}}$ by

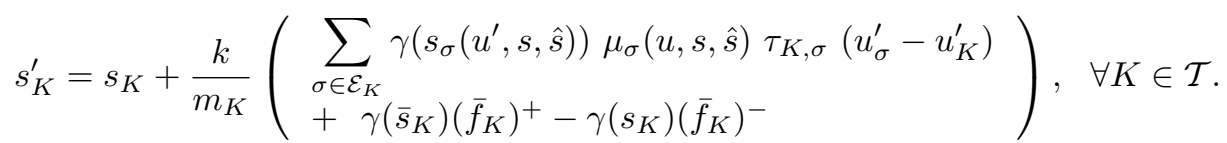

Since $\lambda \geq \alpha$, one has, using (13), that $u^{\prime} \in E$. Then, in order to prove that $\mathcal{A}(u, s) \in E \times L_{\mathcal{D}}(\Omega,[0,1])$, we only have to prove that we can choose $k>0$ such that, for all $K \in \mathcal{T}, 0 \leq s_{K}^{\prime} \leq 1$ (the operator $\mathcal{A}$ is then defined with this value of $k$ ). Using (13), we get

$$
\left|u_{\sigma}^{\prime}-u_{K}^{\prime}\right| \leq\left(\frac{\operatorname{diam}(\Omega)\|f\|_{L^{2}(\Omega)}}{\alpha \inf _{K \in \mathcal{T}, \sigma \in \mathcal{E}_{K}} \tau_{K, \sigma}}\right)^{1 / 2}, \forall K \in \mathcal{T}, \forall \sigma \in \mathcal{E}_{K} .
$$

Denoting by $M_{d u}$ the right hand side of inequality (58), we then take $k>0$ such that

$$
k \leq \inf _{K \in \mathcal{T}} \frac{m_{K}}{L_{\gamma}\left(\beta M_{d u} \sum_{\sigma \in \mathcal{E}_{K}} \tau_{K, \sigma}+\left|\bar{f}_{K}\right|\right)}
$$

(recall that $L_{\gamma}$ is a Lipschitz constant for $\gamma$ ). With such a choice for $k$, we can now prove that for all $K \in \mathcal{T}, s_{K}^{\prime} \in[0,1]$. Indeed, for $K \in \mathcal{T}$, let us multiply (11) (in which we set $\mu=\mu(u, s, \hat{s})$ and $f=\bar{f}$ ) by $\gamma\left(s_{K}\right)$ and substract the result from (57). We then get

$$
s_{K}^{\prime}=s_{K}+\frac{k}{m_{K}}\left(\sum_{\sigma \in \mathcal{E}_{K}} T_{K, \sigma}\left(s_{\sigma}\left(u^{\prime}, s, \hat{s}\right)-s_{K}\right)+T_{K}\left(\bar{s}_{K}-s_{K}\right)\right),
$$

where we define the nonnegative values $T_{K, \sigma}$ and $T_{K}$ by

$$
\begin{cases}T_{K, \sigma}=\frac{\gamma\left(s_{\sigma}\left(u^{\prime}, s, \hat{s}\right)\right)-\gamma\left(s_{K}\right)}{s_{\sigma}\left(u^{\prime}, s, \hat{s}\right)-s_{K}} \mu_{\sigma}(u, s, \hat{s}) \tau_{K, \sigma}\left(u_{\sigma}^{\prime}-u_{K}^{\prime}\right)^{+} & \text {if } s_{\sigma}\left(u^{\prime}, s, \hat{s}\right) \neq s_{K} \\ T_{K, \sigma}=L_{\gamma} \mu_{\sigma}(u, s, \hat{s}) \tau_{K, \sigma}\left(u_{\sigma}^{\prime}-u_{K}^{\prime}\right)^{+} & \text {if } s_{\sigma}\left(u^{\prime}, s, \hat{s}\right)=s_{K}\end{cases}
$$

and

$$
\begin{cases}T_{K}=\frac{\gamma\left(\bar{s}_{K}\right)-\gamma\left(s_{K}\right)}{\bar{s}_{K}-s_{K}}\left(\bar{f}_{K}\right)^{-} & \text {if } \bar{s}_{K} \neq s_{K} \\ T_{K}=L_{\gamma}\left(f_{K}\right)^{-} & \text {if } \bar{s}_{K}=s_{K} .\end{cases}
$$

Then, for $k>0$ verifying (59), we have

$$
0 \leq 1-\frac{k}{m_{K}}\left(\sum_{\sigma \in \mathcal{E}_{K}} T_{K, \sigma}+T_{K}\right),
$$


which ensures that $s_{K}^{\prime}$ is a convex combination of $\left(s_{K}\right)_{K \in \mathcal{T}},\left(\hat{s}_{\sigma}\right)_{\sigma \in \mathcal{E}_{\text {ext }}}$ and $\left(\bar{s}_{K}\right)_{K \in \mathcal{T}}$. This proves that $0 \leq s_{K}^{\prime} \leq 1$.

Since $\mathcal{A}$ is continuous, we can apply Brouwer's fixed point theorem. This gives the existence of $(u, s) \in$ $E \times L_{\mathcal{D}}(\Omega,[0,1])$ such that $\mathcal{A}(u, s)=(u, s)$, which proves the existence of a solution to (52)-(56).

\subsubsection{Convergence of the scheme}

We have the following result, which appears to be very weak compared to the initial ambition of approximating Problem (50).

Theorem 3 Let us assume Hypotheses $(H)$. Let $\left(\mathcal{D}_{n}\right)_{n \in \mathbb{N}}$ be a sequence such that, for all $n \in \mathbb{N}, \mathcal{D}_{n}$ is an admissible discretization of $\Omega$ in the sense of Definition 1 , and $\lim _{n \rightarrow \infty} \operatorname{size}\left(\mathcal{D}_{n}\right)=0$.

Then there exists a subsequence of $\left(\mathcal{D}_{n}\right)_{n \in \mathbb{N}}$, again denoted $\left(\mathcal{D}_{n}\right)_{n \in \mathbb{N}}$, such that, denoting for all $n \in \mathbb{N}$, $\left(u_{n}, s_{n}, \mu_{n}\right) \in H_{\mathcal{D}_{n}}(\Omega) \times L_{\mathcal{D}_{n}}(\Omega,[0,1]) \times \mathcal{M}_{\mathcal{D}_{n}}(\alpha, \beta)$ the solution given by the scheme (52)-(56) with $\mathcal{D}=\mathcal{D}_{n}$, we have:

- the sequence $\left(\mathcal{D}_{n}, \mu_{n}\right)_{n \in \mathbb{N}} H d$-converges in the sense of Theorem 2 to a measurable function $M \in$ $\mathcal{M}(\alpha, \beta, \Omega)$, which implies that $u_{n}$ converges to $\bar{u}=\mathcal{F}(\bar{f}, M)$ in $L^{2}(\Omega)$ as $n \longrightarrow \infty$,

- there exists a function $s \in L^{\infty}(\Omega)$, with $0 \leq s \leq 1$ a.e. such that the sequence $\left(s_{n}\right)_{n \in \mathbb{N}}$ converges to $s$ for the weak $\star$ topology of $L^{\infty}(\Omega)$ and there exists a function $\bar{\gamma} \in L^{\infty}(\Omega)$, with $0 \leq \bar{\gamma} \leq 1$ a.e. such that the sequence $\left(\gamma\left(s_{n}\right)\right)_{n \in \mathbb{N}}$ converges to $\bar{\gamma}$ for the weak $\star$ topology of $L^{\infty}(\Omega)$.

The first item of the conclusion of Theorem 3 is a direct consequence of Theorem 2. The second item is a consequence of the sequential weak $\star$ compactness of the closed balls of $L^{\infty}$. Note that, since the way to handle the convergence of (56) does not seem to be clear, no relation is given in the previous theorem between the limit of $\left(\gamma\left(s_{n}\right) \lambda\left(s_{n}\right)\right)_{n \in \mathbb{N}}$, which is a possibly degenerate diffusion if we consider the second equation of (50) as an elliptic equation on $u$, and the Hd-limit of $\left(\mathcal{D}_{n}, \mu_{n}\right)_{n \in \mathbb{N}}$. Such a relation can be found in the following particular case, where there exists a nondecreasing Lipschitz continuous function $k_{1}:[0,1] \longrightarrow \mathbb{R}$, with $k_{1}(0)=0$ and $k_{1}(1)>0$, and a real $\Lambda \in(0,1)$ such that:

$$
\begin{aligned}
\gamma(s) & =\frac{k_{1}(s)}{k_{1}(s)+\Lambda\left(k_{1}(1)-k_{1}(s)\right)}, \\
\lambda(s) & =k_{1}(s)+\Lambda\left(k_{1}(1)-k_{1}(s)\right), \quad \forall s \in[0,1] .
\end{aligned}
$$

Note that we can take in this case $\beta=k_{1}(1)$ and $\alpha=\Lambda k_{1}(1)$. This particular case corresponds to a mobility of the second phase defined by the function $\Lambda\left(k_{1}(1)-k_{1}().\right)$ (this can be acceptable in some physical situations; recall that $k_{1}$ is the mobility of the first phase). We can then give the following result, which is more complete than Theorem 3 (as previously mentioned, the following theorem does not give the limit of the scheme as a solution of (50) since we could only obtain such a result within a strong convergence property for $\left.\left(s_{n}\right)_{n \in \mathbb{N}}\right)$.

Theorem 4 Let us assume Hypotheses $(H)$ in the particular case (60). Let $\left(\mathcal{D}_{n}\right)_{n \in \mathbb{N}}$ be a sequence such that, for all $n \in \mathbb{N}, \mathcal{D}_{n}$ is an admissible discretization of $\Omega$ in the sense of Definition 1, and $\lim _{n \longrightarrow \infty} \operatorname{size}\left(\mathcal{D}_{n}\right)=0$.

Then there exists a subsequence of $\left(\mathcal{D}_{n}\right)_{n \in \mathbb{N}}$, again denoted $\left(\mathcal{D}_{n}\right)_{n \in \mathbb{N}}$ such that, denoting for all $n \in \mathbb{N}$, $\left(u_{n}, s_{n}, \mu_{n}\right) \in H_{\mathcal{D}_{n}}(\Omega) \times L_{\mathcal{D}_{n}}(\Omega,[0,1]) \times \mathcal{M}_{\mathcal{D}_{n}}\left(\Lambda k_{1}(1), k_{1}(1)\right)$ the solution given by the scheme (52)-(56) with $\mathcal{D}=\mathcal{D}_{n}$, we have, in addition to the conclusions of Theorem 3, the existence of a function $\bar{\gamma} \in L^{\infty}(\Omega)$, with $0 \leq \bar{\gamma} \leq 1$ a.e. such that the sequence $\left(\gamma\left(s_{n}\right)\right)_{n \in \mathbb{N}}$ converges to $\bar{\gamma}$ for the weak $\star$ topology of $L^{\infty}(\Omega)$ and 


$$
\begin{aligned}
& \int_{\Omega} \frac{1}{1-\Lambda}\left(M(x)-\Lambda k_{1}(1) I_{N}\right) \nabla \bar{u}(x) \cdot \nabla \bar{v}(x) d x=\int_{\Omega}\left(\gamma(\bar{s}(x))(\bar{f}(x))^{+}-\bar{\gamma}(x)(\bar{f}(x))^{-}\right) \bar{v}(x) d x, \\
& \forall \bar{v} \in H_{0}^{1}(\Omega) .
\end{aligned}
$$

Note that $k_{1}(\cdot)=\lambda(\cdot) \gamma(\cdot)=\frac{1}{1-\Lambda}\left(\lambda(\cdot)-\Lambda k_{1}(1)\right)$.

Proof We only have to prove (61). Let us assume the hypotheses of Theorem 4. Let $\varphi \in C_{c}^{\infty}(\Omega)$. We define, for $n \in \mathbb{N}$ and omitting indexes $n$ in the right hand sides,

$$
D_{n}=\sum_{K \in \mathcal{T}} \sum_{\sigma \in \mathcal{E}_{K}} \mu_{\sigma} \tau_{K, \sigma}\left(u_{\sigma}-u_{K}\right)\left(\varphi\left(y_{\sigma}\right)-\varphi\left(x_{K}\right)\right),
$$

and

$$
E_{n}=\sum_{K \in \mathcal{T}} \sum_{\sigma \in \mathcal{E}_{K}} \tau_{K, \sigma}\left(u_{\sigma}-u_{K}\right)\left(\varphi\left(y_{\sigma}\right)-\varphi\left(x_{K}\right)\right) .
$$

We have, using results of Theorem 2 ,

$$
\lim _{n \longrightarrow \infty} D_{n}=\int_{\Omega} M(x) \nabla \bar{u}(x) \cdot \nabla \varphi(x) d x .
$$

On the other hand, we have

$$
\lim _{n \longrightarrow \infty} E_{n}=\int_{\Omega} \nabla \bar{u}(x) \cdot \nabla \varphi(x) d x .
$$

Since we assume the particular case (60), we get that

$$
\sum_{K \in \mathcal{T}} \sum_{\sigma \in \mathcal{E}_{K}} \gamma\left(s_{\sigma}(u, s, \hat{s})\right) \mu_{\sigma}(u, s, \hat{s}) \tau_{K, \sigma}\left(u_{\sigma}-u_{K}\right)\left(\varphi\left(y_{\sigma}\right)-\varphi\left(x_{K}\right)\right)=\frac{1}{1-\Lambda}\left(D_{n}-\Lambda k_{1}(1) E_{n}\right) .
$$

Using the fact that $\sum_{K \in \mathcal{T}} \gamma\left(s_{K}\right)\left(\bar{f}_{K}\right)^{-} \varphi\left(x_{K}\right) \longrightarrow \int_{\Omega} \bar{\gamma}(x)(\bar{f}(x))^{-} \varphi(x) d x$ as $n \longrightarrow \infty$ (thanks to the $L^{\infty}$ weak $\star$ convergence of $\gamma\left(s_{n}\right)$ to $\left.\bar{\gamma}\right)$, we thus get (61) with $\varphi \in C_{c}^{\infty}(\Omega)$. Then we obtain (61) using a classical result of density.

\section{Concluding remarks}

The notion of Hd-convergence, developed in this paper, gives a useful tool for studying the convergence of a discrete finite volume scheme, used for the approximation of a two-phase flow in a porous medium. The proof of the Hd-convergence theorem mimics that of the H-convergence theorem; however, although the methods are similar, the limits can be different. This discrete tool is therefore adapted to the case of a coupled discretization: the discrete pressure field is solution of a discrete scheme for an elliptic equation, the coefficients of which result from another discrete scheme in the same grid.

This tool thus helps to get the limit problem of which the limit of the approximate pressure is solution. A weak limit also exists for the saturation since the discrete values are bounded as well as the continuous ones. Unfortunately, we are not able to link the Hd-limit of the sequence of discrete total mobilities and a convenient limit of the sequence of saturations.

Finally, the time-dependent must now be studied. Following [18] in which the G-convergence notion is adapted to general parabolic time-dependent operators, it is then possible to develop a discrete $\mathrm{H}$ convergence in the case of a two-phase flow in compressible porous media (see [8]). 


\section{References}

[1] Alt, H.W., Luckhaus, S., Visintin, A. On nonstationary flow through porous media, Ann. Mat. Pura. Appl., 136 (1984), 303-316.

[2] Alt, H.W., Di Benedetto, E. Nonsteady flow of water and oil through inhomogeneous porous media, Ann. Scuola Norm. Sup. Pisa, 12 (1985), 335-392.

[3] Aziz, K, Settari, A., Petroleum Reservoir Simulation, Applied Science (1979), London.

[4] Carrillo, J. Entropy solutions for nonlinear degenerate problems, Arch. Rat. Mech. Anal., 147 (1999), 269-361.

[5] Chen, Z. Degenerate Two-Phase Incompressible Flow, J. of Differential Equations, 171 (2001), 203-232.

[6] Droniou, J., Gallouët, T. Finite volume methods for convection-diffusion equations with righthand side in $H^{-1}$, accepted for publication in $M^{2} A N,(2002)$.

[7] Eymard, R., Gallouët, T. Convergence d'un schéma de type éléments finis-volumes finis pour un système formé d'une équation elliptique et d'une équation hyperbolique, $M^{2} A N, 27$ (1993), 843-861.

[8] Eymard, R., Gallouët, T. Finite volume schemes and the viscous fingering problem, in preparation (2002).

[9] Eymard, R., Gallouët, T., Herbin, R. Finite Volume Methods, Handbook of Numerical Analysis, P.G. Ciarlet and J.L. Lions. eds., VII (2000) 723-1020.

[10] Eymard, R., Gallouët, T., Herbin, R., Michel, A. Convergence of a finite volume scheme for nonlinear degenerate parabolic equations accepted for publication in Num. Math. (2001).

[11] Gagneux, G., Madaune-Tort, M. Analyse mathématique de modèles non linéaires de l'ingénierie pétrolière, Mathématiques et Applications 22, Springer-Verlag (1996).

[12] KozLov, S.M., Averaging of difference schemes, Math. USSR Sb 57 (1987), 351-369.

[13] Krushkov, S.N. First order quasilinear equations with several space variables, Math. USSR. Sb. 10 (1970), 217-243.

[14] Murat, F., H-convergence, Séminaire d'analyse fonctionnelle et numérique de l'Université d'Alger (1977-1978).

[15] Ohlberger, M., Convergence of a mixed finite element-finite volume method for the two phase flow in porous media, East-West J. Numer. Math. 5 (1997), 183-210.

[16] Piatnitski, A. RÉmy, E., Homogenization of elliptic difference operators, Siam J. Math. Anal. 33, 1 (2001), 53-83.

[17] Spagnolo, S., Sulla convergenza di soluzioni di equazioni paraboliche ed ellittiche, Ann. Scuola Norm. Sup. Pisa, Sci. Fis. Mat., III. Ser. 22 (1968), 571-597.

[18] Svanstedt, N., G-convergence of parabolic operators, Nonlinear Anal., Theory Methods Appl. 7 (1999) 807-842.

[19] Tartar, L., Cours Peccot, Collège de France (Paris, 1977).

[20] Tartar, L. Homogénéisation et H-mesures. (Homogenization and H-measures). (French) ESAIM, Proc. 6 (1999) 111-131.

[21] Vignal, M.H., Convergence of a finite volume scheme for an elliptic-hyperbolic system, $M^{2} A N, \mathbf{3 0}$, (1996), 841-872. 